\title{
Southern annular mode dynamics in observations and models, Part II: Eddy feedbacks
}

Article

Published Version

Simpson, I. R., Shepherd, T. G., Hitchcock, P. and Scinocca, J. F. (2013) Southern annular mode dynamics in observations and models, Part II: Eddy feedbacks. Journal of Climate, 26 (14). pp. 5220-5241. ISSN 1520-0442 doi:

https://doi.org/10.1175/JCLI-D-12-00495.1 Available at https://centaur.reading.ac.uk/33006/

It is advisable to refer to the publisher's version if you intend to cite from the work. See Guidance on citing.

Published version at: http://dx.doi.org/10.1175/JCLI-D-12-00495.1

To link to this article DOI: http://dx.doi.org/10.1175/JCLI-D-12-00495.1

Publisher: American Meteorological Society

All outputs in CentAUR are protected by Intellectual Property Rights law, including copyright law. Copyright and IPR is retained by the creators or other copyright holders. Terms and conditions for use of this material are defined in the End User Agreement.

www.reading.ac.uk/centaur 
Central Archive at the University of Reading

Reading's research outputs online 


\title{
Southern Annular Mode Dynamics in Observations and Models. Part II: Eddy Feedbacks
}

\author{
ISLA R. SIMPSON \\ Department of Physics, University of Toronto, Toronto, Ontario, Canada, and Division of Ocean and Climate Physics, \\ Lamont-Doherty Earth Observatory, Columbia University, Palisades, New York \\ THEODORE G. SHEPHERD \\ Department of Physics, University of Toronto, Toronto, Ontario, Canada, and Department of Meteorology, \\ University of Reading, Reading, United Kingdom \\ PETER HitCHCOCK* \\ Department of Physics, University of Toronto, Toronto, Ontario, Canada \\ JOHN F. SCINOCCA \\ Canadian Centre for Climate Modelling and Analysis, Environment Canada, Victoria, British Columbia, Canada
}

(Manuscript received 26 July 2012, in final form 7 February 2013)

\begin{abstract}
Many global climate models (GCMs) have trouble simulating southern annular mode (SAM) variability correctly, particularly in the Southern Hemisphere summer season where it tends to be too persistent. In this two-part study, a suite of experiments with the Canadian Middle Atmosphere Model (CMAM) is analyzed to improve the understanding of the dynamics of SAM variability and its deficiencies in GCMs. Here, an examination of the eddy-mean flow feedbacks is presented by quantification of the feedback strength as a function of zonal scale and season using a new methodology that accounts for intraseasonal forcing of the SAM.

In the observed atmosphere, in the summer season, a strong negative feedback by planetary-scale waves, in particular zonal wavenumber 3 , is found in a localized region in the southwest Pacific. It cancels a large proportion of the positive feedback by synoptic- and smaller-scale eddies in the zonal mean, resulting in a very weak overall eddy feedback on the SAM. CMAM is deficient in this negative feedback by planetary-scale waves, making a substantial contribution to its bias in summertime SAM persistence. Furthermore, this bias is not alleviated by artificially improving the climatological circulation, suggesting that climatological circulation biases are not the cause of the planetary wave feedback deficiency in the model.

Analysis of the summertime eddy feedbacks in the models from phase 5 of the Coupled Model Intercomparison Project (CMIP5) confirms that this is indeed a common problem among GCMs, suggesting that understanding this planetary wave feedback and the reason for its deficiency in GCMs is key to improving the fidelity of simulated SAM variability in the summer season.
\end{abstract}

\footnotetext{
* Current affiliation: Department of Applied Mathematics and Theoretical Physics, University of Cambridge, Cambridge, United Kingdom.
}

Corresponding author address: Isla R. Simpson, Division of Ocean and Climate Physics, Lamont-Doherty Earth Observatory, Columbia University, P.O. Box 100, Route 9W, Palisades, NY 10965-1000.

E-mail: isla@ldeo.columbia.edu

\section{Introduction}

To accurately simulate future changes to the climate system we must be certain that global climate models (GCMs) are accurately simulating the relevant dynamical processes in the atmospheric circulation. One way to do this is to ensure that GCMs are able to simulate present-day natural variability in the atmospheric circulation well, and for the correct reasons. At present,

DOI: 10.1175/JCLI-D-12-00495.1 
GCMs do not generally exhibit the correct time scale of variability in the Southern Hemisphere ( $\mathrm{SH}$ ) midlatitudes, particularly in the summer season.

The southern annular mode (SAM) is the dominant mode of variability in the $\mathrm{SH}$ tropospheric midlatitude circulation (Kidson 1988; Thompson and Wallace 2000) and is typically described using the first empirical orthogonal function (EOF) of zonal mean zonal wind or geopotential height. These EOFs are characterized by a dipolar structure of zonal mean zonal wind anomalies centered on the eddy-driven midlatitude jet, contributing to latitudinal migrations of the tropospheric westerly winds. A relevant characteristic of the SAM is its temporal persistence, as this provides information on the relative importance of feedback and dissipative processes acting on the SAM zonal wind anomalies. If this is not simulated correctly, then it may imply a bias in the dynamics of midlatitude jet migrations in the models. Currently GCMs, both coupled and uncoupled, exhibit a SAM that is much too persistent in the summer season (Gerber et al. 2008, 2010).

The fluctuation-dissipation theorem (Leith 1975) predicts that the magnitude of response to a forcing will be proportional to the time scale of natural unforced variability. In the context of the SAM, the physical explanation behind this lies in the fact that both the time scale of the SAM and the magnitude of a forced SAM-like response are influenced by the same feedback and dissipative processes. Thus, if the models do not get the SAM time scale correct, we should be concerned as to whether they will simulate the magnitude of forced responses correctly. Since many climate forcings result in a latitudinal shift of the $\mathrm{SH}$ jet that projects strongly onto the SAM, we must understand the cause of this bias and alleviate it in order to have confidence in future climate projections.

In the first part of this study (Simpson et al. 2013, hereafter Part I), a suite of experiments with the Canadian Middle Atmosphere Model (CMAM) were analyzed with an aim to improve our understanding of this common model problem. By systematically improving the representation of stratospheric variability and improving the climatological tropospheric $3 \mathrm{D}$ circulation it was possible to demonstrate that the SAM time scale bias remains in CMAM even when the representation of both these aspects are improved. These results suggest that while the common model bias in climatological jet position may be a contributing factor to the bias in SAM time scale (Kidston and Gerber 2010; Arakelian and Codron 2012), particularly in models that exhibit a fairly severe bias in jet position, it is unlikely to be the only factor. This is certainly the case in CMAM where the bias is not alleviated by improving the climatological winds. Furthermore, this suggests that other biases in tropospheric climatology (e.g., jet strength or degree of zonality) are also not the explanation of the overly persistent SAM variability. Part I has also revealed that a substantial proportion of the SAM time scale bias arises from processes that may be considered "internal" to the tropospheric dynamics since the bias still occurs when sources of "external" intraseasonal forcing on the SAM, such as stratospheric variability or sea surface temperature (SST) variability, are removed. The aim of the current study is then to improve our understanding of the cause of this bias in internal tropospheric jet dynamics.

The studies of Robinson (1996, 2000) and Lorenz and Hartmann $(2001,2003)$ demonstrated the key role for feedbacks between tropospheric eddy momentum flux anomalies and zonal mean flow anomalies in the dynamics of the annular modes. Lorenz and Hartmann (2001, hereafter LH01) identified the importance of these feedbacks in governing the temporal persistence of the SAM and found that SAM persistence in the observed atmosphere could be well explained by the balance between a positive feedback on the zonal wind anomalies by high-frequency eddies, a negative feedback by lowerfrequency eddies, and the dissipation of zonal wind anomalies by surface friction. Various different components of the feedback process have been established (Robinson 1996, 2000; LH01; Kidston et al. 2010) and there is little doubt that the strength of eddy-mean flow feedbacks is important for SAM persistence. Here, we assess whether a deficiency in eddy feedbacks is contributing to the summertime bias in SAM persistence in CMAM and in other models in the archive of phase 5 of the Coupled Model Intercomparison Project (CMIP5).

The CMAM model experiments are described in detail in Part I so only a brief overview is given in section 2, followed by a description of diagnostics in section 3 . In section 4 an analysis of the eddy feedbacks on the SAM in the observed atmosphere and in CMAM is presented. This includes the development of a method to quantify eddy feedback strength in the presence of intraseasonal forcing of the SAM (e.g., that which arises from the presence of stratospheric variability). This analysis begins by examination of the eddy feedbacks in the December-February (DJF) season, which is when the bias is most severe, and then proceeds to examine the seasonal variation in eddy feedbacks. By quantifying the eddy feedback strength as a function of season and examining the contribution from different zonal scales, a more detailed understanding of the dynamics of SAM variability in the observed atmosphere is obtained. In particular, planetary-scale waves are found to provide a negative feedback on the SAM in the summer season. 
TABLE 1. Summary of model experiments. Columns indicate the name of the experiment, the length, whether the tropospheric climatological circulation is biased or whether it is bias corrected, whether the timing of the vortex breakdown date is too late or whether that is bias corrected, whether zonal mean stratospheric variability is present, and the SSTs prescribed at the lower boundary.

\begin{tabular}{llllll}
\hline \hline Name & \multicolumn{1}{c}{ Length } & Troposphere & Stratosphere & Stratospheric variability & SSTs \\
\hline FREE & $100 \mathrm{yr}$ & Biased & Biased & Yes & Climatological \\
NUDG & $100 \mathrm{yr}$ & Biased & Biased & No & Climatological \\
BC & $2 \times 1970-2009$ & Corrected & Corrected & Yes & Time varying \\
BCNUDG & $100 \mathrm{yr}$ & Corrected & Corrected & No & Climatological \\
\hline
\end{tabular}

CMAM is deficient in this planetary wave feedback and so section 5 proceeds to examine the details of this planetary wave feedback further. The relevance of these results for other models in the CMIP5 archive is then assessed in section 6, discussion is provided in section 7, and conclusions are drawn in section 8 .

\section{Model experiments and data}

The Canadian Middle Atmosphere Model is a comprehensive, stratosphere-resolving GCM with 71 levels in the vertical stretching from the surface to $0.0006 \mathrm{hPa}$ $(\sim 100 \mathrm{~km})$ (Scinocca et al. 2008). This version is run without interactive chemistry and with prescribed seasonally varying SSTs. A series of experiments are performed that employ nudging, bias correction, or both. Nudging involves the relaxation of the model vorticity, divergence, and temperature toward a specified state whereas bias correction involves the application of a constant tendency to these fields that varies only with season. Because there is no relaxation applied in bias correction, there is no damping of variability.

Four different CMAM experiments are used (summarized in Table 1 and described in more detail in Part I). FREE is a free-running control simulation with climatological SSTs. NUDG employs nudging on the zonal mean in the stratosphere toward the seasonally varying climatology of FREE so as to eliminate zonal mean stratospheric variability but keep the same climatological circulation as FREE. For brevity, we will often refer to this zonal mean stratospheric variability as simply "stratospheric variability." In BC and BCNUDG bias correction is applied in the troposphere on scales down to total horizontal wavenumber $n=21$ in order to improve the climatological tropospheric jet structure and stationary circulation patterns. It should be emphasized that this improves not only the zonal mean but also the full 3D climatological circulation. The result of the bias correction process is an improvement in the climatology (as demonstrated in Part I), but the difference from the nudging process is that variability still occurs around that improved climatology. In BC, bias correction is also applied in the stratosphere to improve the timing of the breakdown of the polar vortex, and in BCNUDG the zonal mean in the stratosphere is nudged toward the European Centre for Medium-Range Weather Forecasts (ECMWF) Interim Re-Analysis (ERA-Interim; Dee et al. 2011) climatology so as to have the correct timing of the vortex breakdown but no stratospheric variability. These four experiments will be compared with ERA-Interim from 1979 to 2010, which was obtained at 18 vertical levels from the surface to $10 \mathrm{hPa}$ and interpolated onto the same horizontal and vertical grid as the CMAM data using cubic splines.

Daily means are used and in the case of any zonal mean flux terms, the 6-hourly instantaneous fluxes were first calculated before the daily mean was obtained. Kuroda and Mukougawa (2011) recently demonstrated an important role for high-frequency, medium-scale waves (i.e., smaller than synoptic scales) in contributing to the eddy feedback on the SAM. We also find this to be the case and emphasize that it is important that the small-scale eddy momentum flux is obtained using instantaneous values of $u$ and $v$ rather than, for example, daily means. When examining the spatial structure of eddy momentum fluxes by large-scale waves in section 5 , daily mean $u$ and $v$ are used to calculate those eddy fluxes.

In section 6 the CMAM results are compared with models from the CMIP5 multimodel ensemble. The analysis is performed using 6-hourly $u$ and $v$ for the historical simulation from 1950 to 2005 on the 250-, 500-, and $850-\mathrm{hPa}$ pressure levels. Monthly-mean $300-\mathrm{hPa}$ geopotential height is also shown. Only models for which 6-hourly $u$ and $v$ were available at the time of writing are used.

\section{Diagnostics}

In Part I the SAM was described by the first EOF of zonal mean geopotential on each vertical level. Here, to be consistent with the existing literature on tropospheric eddy feedbacks (LH01; Lorenz and Hartmann 2003; Kidston et al. 2010) the SAM will now be described by the first EOF of vertically averaged zonal mean zonal wind from $100 \mathrm{hPa}$ to the surface. The pressure weighted 


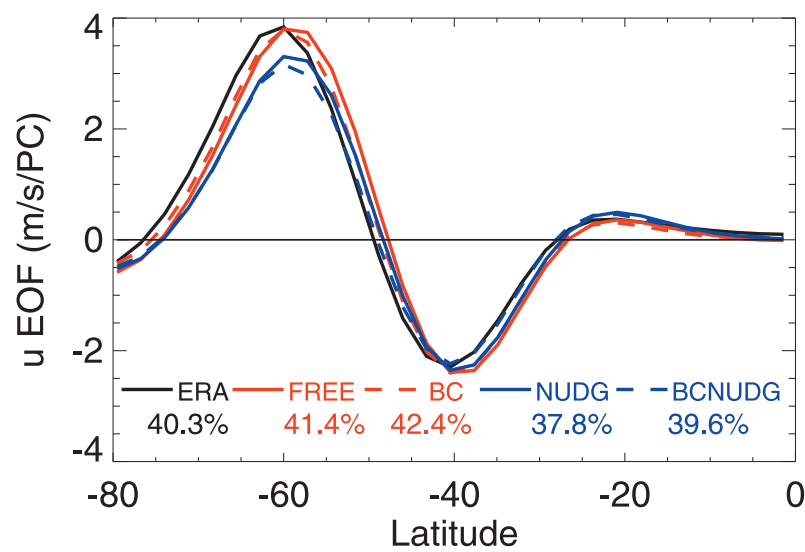

FIG. 1. Structure of the first EOF of vertically averaged zonal wind for each of the experiments and ERA-Interim. The percentage of variance explained by the first EOF is indicated in the legend.

vertical average of a given zonally averaged field $\bar{x}$ will be denoted $[\bar{x}]$. The SAM is described by the first EOF of vertically averaged zonal wind $[\bar{u}]$ after deseasonalizing (and linear detrending in the case of the reanalysis and CMIP5) following the weighting procedure of Baldwin et al. (2009), with the exception that the EOF e and principal component time series or SAM index $\mathrm{PC}(t)$ are defined such that $\mathrm{PC}(t)$ has unit variance and the EOF structure has units of $\mathrm{m} \mathrm{s}^{-1} \mathrm{PC}^{-1}$. Thus, the vertically integrated zonal wind anomaly associated with the SAM is given by $[\bar{u}]_{s}(\phi, t)=\mathrm{PC}(t) \mathbf{e}(\phi)$. Note that $\mathbf{e}$ and $\mathrm{PC}$ are defined using all months of all years but the same conclusions can be drawn if they are defined separately for each individual season. The structure of this EOF and the percentage variance explained by it are shown in Fig. 1 for each CMAM experiment and ERAInterim. This same color scheme will be used throughout the paper: black for ERA-Interim, red for the CMAM simulations that have stratospheric variability, blue for those that do not, and dashed lines for the CMAM simulations where bias correction is applied. The EOF structure is similar and explains a similar fraction of the variance (around $40 \%$ ) in each situation. It represents the same mode of variability as was captured by the geopotential height EOF in Part I (i.e., a dipolar change in zonal wind centered on the mean midlatitude westerly jet position). The runs with a nudged stratosphere have a slightly smaller amplitude of variability, even though the stratosphere is only nudged down to the $64-\mathrm{hPa}$ level. Bias correction shifts the location of the maximum and minimum in the structure slightly poleward.

To examine the contributions to the maintenance of SAM anomalies, the various terms in the Eulerian zonal mean momentum budget equation are vertically averaged and projected onto this EOF pattern to give the forcing of SAM anomalies by each term; that is, the forcing of the SAM by a term $[\bar{x}]$ is given by

$$
[\bar{x}]_{S}(t)=\frac{[\overline{\mathbf{x}}](t) \mathbf{W e}}{\sqrt{\mathbf{e}^{\mathrm{T}} \mathbf{W e}}},
$$

where $[\bar{x}]_{s}$ denotes the component of the field $[\bar{x}]$ that projects onto the latitudinal structure of the SAM, $[\overline{\mathbf{x}}](t)$ is a vector form of $[\bar{x}](\phi, t)$ with latitude dimensions, and $\mathbf{W}$ is a matrix with diagonal elements equal to the $\cos$ (latitude) weighting used when defining the EOF e (Baldwin et al. 2009). Thus, the vertically averaged Eulerian zonal mean momentum budget in spherical coordinates in terms of quantities projected onto the SAM is

$$
\begin{aligned}
\frac{\partial[\bar{u}]_{S}}{\partial t}= & {[f \bar{v}]_{S}-\left[\frac{\bar{v}}{a \cos \phi} \frac{\partial \bar{u} \cos \phi}{\partial \phi}\right]_{S}-\left[\bar{\omega} \frac{\partial \bar{u}}{\partial p}\right]_{S} } \\
& -\left[\frac{1}{a \cos ^{2} \phi} \frac{\partial\left(\overline{u^{\prime} v^{\prime}} \cos ^{2} \phi\right)}{\partial \phi}\right]_{S}-\left[\frac{\partial\left(\overline{\omega^{\prime} u^{\prime}}\right)}{\partial p}\right]_{S}+[\bar{X}]_{S},
\end{aligned}
$$

where $u, v$, and $\omega$ are the zonal, meridional, and vertical (pressure) velocities respectively, overbars denote the zonal mean, $f$ is the Coriolis parameter, and $\bar{X}$ represents the zonal mean tendency associated with parameterized processes such as surface friction. To understand the difference in persistence of $[\bar{u}]_{s}$ anomalies, lagged linear regressions of these projected momentum budget terms onto the SAM index $\mathrm{PC}(t)$ will be analyzed. For the case of, for example, the DJF season, these lagged regressions are performed using the DJF SAM index and a segment of the relevant field of the same length as DJF but displaced forward or backward by the required lag.

\section{Results}

\section{a. Summertime feedbacks}

Since NUDG and BCNUDG were demonstrated in Part I to have a bias that is internal to the troposphere in DJF, we begin with analysis of SAM feedbacks in this season. Figure 2a shows lagged regressions of the SAM zonal wind anomalies $[\bar{u}]_{s}$ onto the SAM index $\operatorname{PC}(t)$. This demonstrates the growth of SAM anomalies at negative lags up to a maximum at lag 0 followed by a decay at positive lags. Small differences in the peak amplitude of SAM anomalies are evident at lag 0 with simulations with stratospheric variability (red) having a larger amplitude than those without (blue) (also seen in Fig. 1). The decay of SAM anomalies at positive lags demonstrates the same difference in SAM persistence 
(a) $[u]_{s}$ regressed onto $P C$

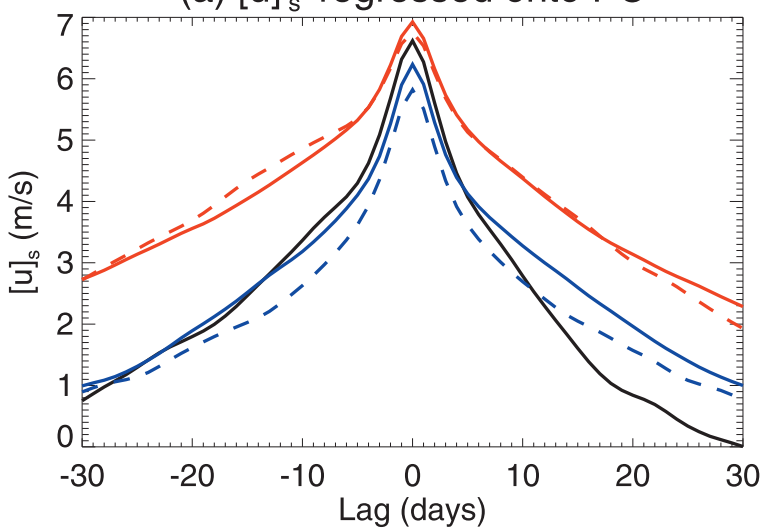

(b) $\mathrm{d}[\mathrm{u}]_{s} / \mathrm{dt}$ regressed onto $\mathrm{PC}$

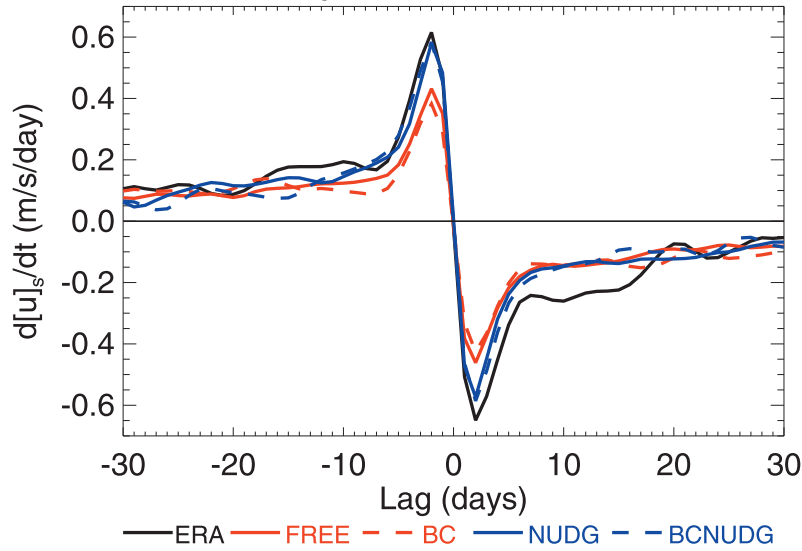

FIG. 2. Lagged regressions of (a) $[\bar{u}]_{s}$ and (b) $\partial[\bar{u}]_{s} / \partial t$ onto $\mathrm{PC}(t)$ for the DJF season.

that was obtained using the time scale of the geopotential height SAM in Part I: SAM zonal wind anomalies decay more rapidly in ERA-Interim than in any of the model simulations. SAM anomalies are most persistent in the two simulations with stratospheric variability and there does not appear to be a notable influence of bias correction on the decay rate of zonal wind anomalies.

Note that in the simulations with stratospheric variability (FREE and BC), and especially in the observations, the $[\bar{u}]_{s}$ regression is not symmetric about lag 0 . This is because the troposphere in DJF transitions from a state in the early part of the season where stratospheric variability substantially enhances the SAM time scale to a state in the later part of the season where stratospheric variability has a much smaller effect (Baldwin et al. 2003; Simpson et al. 2011). Thus, at negative lags, a larger proportion of the regression is computed from a time where stratospheric variability is contributing to enhanced persistence. The regressions are much more symmetric about lag 0 in the simulations without stratospheric variability.
The rate of change of SAM zonal wind anomalies (i.e., the time derivative of Fig. 2a) is shown in Fig. $2 b$ and there are two main points to be noted from this. The first is that when comparing FREE and BC (which have stratospheric variability) to NUDG and BCNUDG (which do not), the main difference is occurring at small positive and negative lags between -5 and +5 days. Comparing the red and the blue curves demonstrates that the simulations with stratospheric variability exhibit a smaller rate of change of zonal wind anomalies at these lags, which causes the $[\bar{u}]_{s}$ regressions in Fig. 2 a to remain much larger out to large lags, yielding longer SAM time scales in these simulations. This may be expected from the presence of a persistent intraseasonal forcing from the stratosphere on the SAM in the simulations with stratospheric variability.

Intuitively one might expect that the presence of a long time scale forcing would alter the regression at large positive lags, as is indeed the case here. But, perhaps less intuitively, it appears that this is being done primarily through an alteration to the rate of change of zonal wind anomalies at small lags. It is the more random fluctuations which are important at small lags, giving rise to the rapid acceleration and deceleration of SAM anomalies around lag 0 . This alteration to the structure of the regression at small lags in the presence of stratospheric variability can be understood as follows. The stratospheric variability provides an effective intraseasonal forcing, and results in the more random SAM fluctuations (which dominate at short lags) becoming relatively less important in the $[\bar{u}]_{s}$ regression, reducing the rate of change at small lags. This is demonstrated using synthetic time series in the appendix. Of course, the reanalysis also has stratospheric variability but its curve in Fig. 2b looks more like the CMAM simulations without stratospheric variability at small positive and negative lags. It may be that in the model, stratospheric variability has too much of an influence on the troposphere, and indeed a substantial influence of stratospheric variability on tropospheric SAM persistence was shown in Part I. This may be because of the bias in tropospheric dynamics, or it may be for some other reason. Understanding the question of the potential role of a stratospheric influence on the SAM time scale bias is beyond the scope of this study. For now, we are concerned with the bias in internal tropospheric dynamics in the model.

In this regard, the second important point to note from Fig. $2 b$ is the obvious difference in the decay rate of SAM anomalies between all the simulations and ERAInterim at positive lags between around +7 and +17 days. At these lags the decay of SAM zonal wind anomalies is very similar in each of the model simulations and this 
(a) Budget terms, NUDG

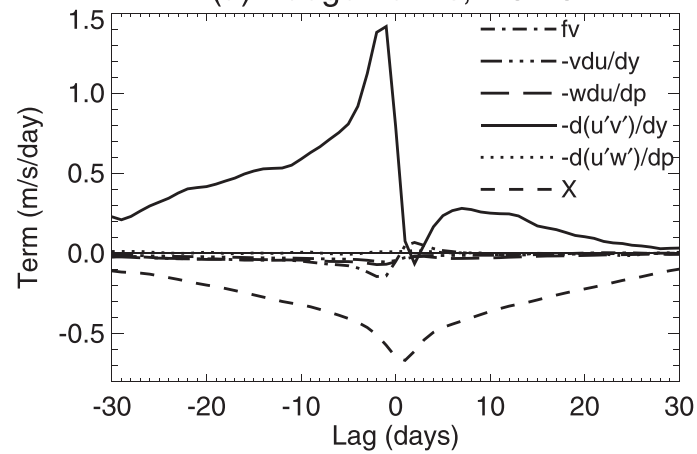

(b) Acceleration

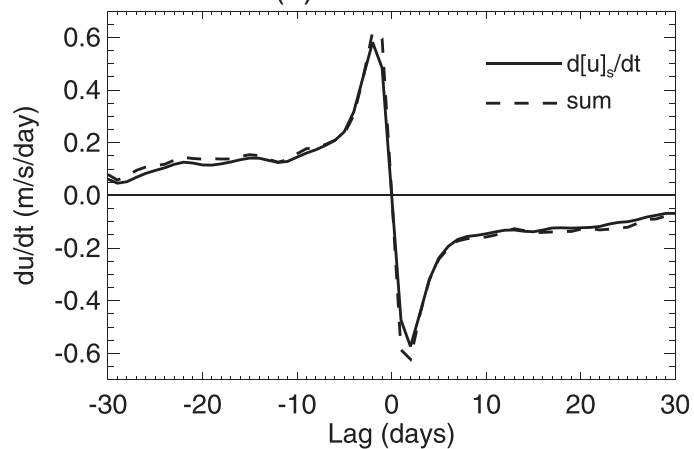

FIG. 3. (a) Each of the momentum budget terms in (2) regressed onto PC $(t)$ for the NUDG experiment for the DJF season. (b) The sum of each of the terms in (a) and $\partial[\bar{u}]_{s} / \partial t$ regressed onto PC $(t)$ for the NUDG experiment.

decay is considerably slower than that in ERA-Interim (a difference of around $0.1 \mathrm{~m} \mathrm{~s}^{-1} \mathrm{day}^{-1}$ ). To understand the cause of the bias in internal tropospheric dynamics that leads to enhanced SAM persistence in the model, we must understand why the rate of decay of SAM zonal wind anomalies differs at these lags and the answer must lie in one of the momentum budget terms in (2).

Figure 3a shows lagged regression onto the SAM index for each of the individual terms on the right-hand side of (2) for the NUDG experiment to demonstrate the dominant terms in the maintenance/decay of SAM anomalies. Figure $3 b$ then confirms that the rate of change of regressed $[\bar{u}]_{s}$ can be explained by the sum of the individual regressed terms on the right-hand side of (2), so the momentum budget of regressed quantities balances well. The eddy momentum flux convergence and friction are by far the dominant terms in the evolution of the SAM anomalies. At positive lags, eddy momentum flux convergence acts to maintain the SAM anomalies whereas friction acts to dissipate them, as was found by LH01. This is true of each of the other simulations and ERA-Interim (although for ERA-Interim $\bar{X}$ cannot be calculated explicitly, only as a residual). Given the importance of the eddy momentum flux convergence in maintaining SAM anomalies and LH01's identification of the importance of this in contributing to SAM persistence, we consider whether a difference in the feedback associated with the eddy momentum flux convergence can explain the difference in the decay of anomalies at lags greater than about 7 days, as seen in Fig. 2b.

LH01 quantified the eddy feedback using cross-spectral analysis. Here, we follow LH01's ideas to some extent but adopt a different method that can account for the presence of external forcing of the SAM on intraseasonal time scales, as discussed in the appendix. The eddy forcing of the SAM $\left[-\left(1 / a \cos ^{2} \phi\right) \partial \overline{u^{\prime} v^{\prime}} \cos ^{2} \phi / \partial \phi\right]_{s}$ will be denoted $m$ for short. LH01 hypothesized that $m$ consists of a random component $\tilde{m}$ that is independent of the preexisting SAM state, and a feedback component $b[\bar{u}]_{S}$ that is linearly related to the SAM zonal wind anomalies (i.e., $m=\tilde{m}+b[\bar{u}]_{s}$ ). We linearly regress $m$ and $[\bar{u}]_{s}$ onto the SAM index such that for a lag $l$, $m(t+l) \approx \beta_{m}(l) \mathrm{PC}(t)$ and $[\bar{u}]_{s}(t+l) \approx \beta_{u_{s}}(l) \mathrm{PC}(t)$, where $\beta_{m}$ and $\beta_{u_{\mathrm{s}}}$ are the regression coefficients. At lag $l$ the eddy forcing of the SAM can be written as $m(t+l)=\tilde{m}(t+l)+b[\bar{u}]_{s}(t+l)$ or, in terms of the regression coefficients, as $\beta_{m}(l) \mathrm{PC}(t)=\beta_{\tilde{m}}(l) \mathrm{PC}(t)+$ $b \beta_{u_{\mathrm{s}}}(l) \mathrm{PC}(t)$. At small lags the regression will include SAM anomalies that are driven by the random eddy forcing $\tilde{m}$. However, at large positive lags, beyond the time scale over which there is significant autocorrelation in $\tilde{m}$, the feedback component of the eddy forcing $b[\bar{u}]_{s}$ will dominate in the $m$ regression (i.e., $\beta_{\tilde{m}}=0$ ). Therefore, at sufficiently large lags it becomes $\beta_{m} \mathrm{PC}(t) \approx$ $b \beta_{u_{s}} \mathrm{PC}(t)$. Thus, taking the ratio of the $m$ and $[\bar{u}]_{s}$ regression coefficients at these lags provides an estimate of $b$ (i.e., $b=\beta_{m} / \beta_{u_{\mathrm{s}}}$ ). See the appendix for more details.

Figure 4 shows the autocorrelation of the eddy forcing of the SAM $m$ divided into synoptic and smaller scales (zonal wavenumber $k>3$ ) and planetary scales ( $k=$ $1-3)$. The autocorrelation of the eddy forcing on each of these scales drops rapidly over a few days to negative values and returns to zero by around 7 days, as was also found by LH01. This portion of the autocorrelation function is assumed to be associated with the random portion of the eddy forcing $\tilde{m}$. At lags greater than 7 days for the total eddy forcing (Fig. 4a) there is also a small positive correlation related to the feedback component, which represents only a fraction of the variance of $m$ but decays on the time scale of the SAM. In the following it will be shown that there is also a significant negative planetary wave feedback on the SAM in the reanalysis, but the fact that it is not visible in Fig. $4 \mathrm{~b}$ suggests that it represents only a small fraction of the planetary eddy variance. At lags of greater than 7 days, 
(a) m autocorrelation ( $k>3$ )

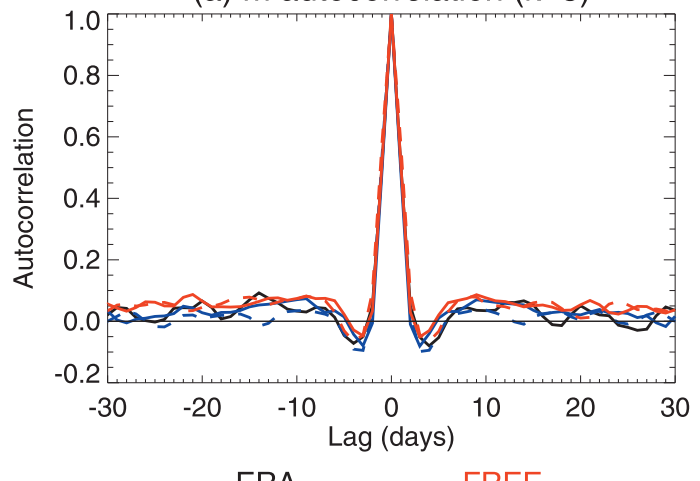

(b) m autocorrelation $(\mathrm{k}=1-3)$

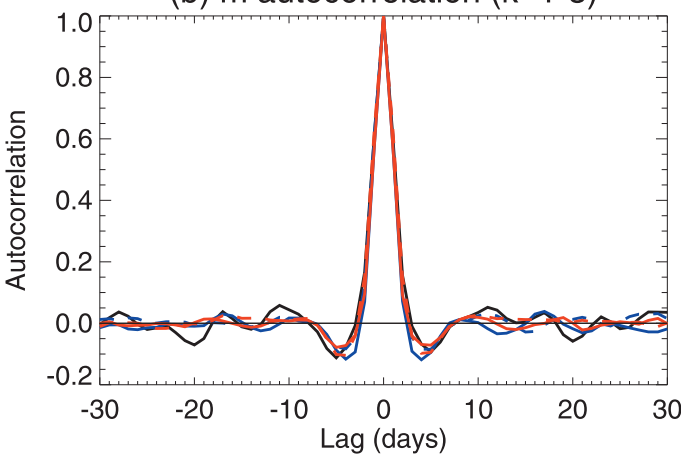

NUDG _ - . BCNUDG

FIG. 4. The lagged autocorrelation of the eddy forcing $m$ for (a) zonal wavenumber $k>3$ and (b) zonal wavenumber $k=1-3$ for the DJF season.

one can then expect that the above estimation of $b$ based on the ratio of the $m$ and $[\bar{u}]_{s}$ regression coefficients should work since this is beyond the autocorrelation time scale of $\tilde{m}$.

On the left-hand side of Fig. 5 the eddy forcing $m$ regressed onto the SAM index (i.e., $\beta_{m}$ ) is shown. On the right-hand side, $b$ is plotted for lags between +7 and +14 days at which time the component due to $\tilde{m}$ will be negligible but the zonal wind anomalies are still substantial. In Fig. 5g, the average feedback strength over lags from 7 to 14 days is quantified with $95 \%$ confidence limits calculated via the method outlined in the appendix.

We begin by examination of the synoptic and smallerscale eddies (zonal wavenumber $k>3$ ) in Figs. 5a,b,g (left). In agreement with LH01 the regression of $m$ onto the SAM index is positive at positive lags indicating a positive feedback by synoptic-scale eddies. The quantification of the feedback strength, $b$, in Fig. 5b shows roughly constant values at each lag for each case, demonstrating that the simple linear model of the feedback works well. Each case has a strong positive feedback by synoptic-scale eddies onto the SAM and the feedback strength in the model compares relatively well with ERAInterim. The feedback strengths also compare relatively well between the simulations with and without stratospheric variability, suggesting that our method of taking into account intraseasonal forcing on the SAM is working. It is interesting to note that there is also some small influence of the bias correction on synoptic feedback strength. The feedback strength is lower in both biascorrected runs, which is consistent with previous work that has related SAM time scales and eddy feedback strength to climatological jet latitude, since the bias correction shifts the jet slightly poleward (see Part I) (Kidston and Gerber 2010; Barnes et al. 2010; Simpson et al. 2012).
In contrast to the synoptic scales, when considering planetary-scale waves $(k=1-3)$ in Figs. 5c,d,g (middle), a negative feedback is found. In ERA-Interim, there is some variability of the feedback strength with lag, which indicates that there may be some limitations to the ability of the linear model to describe the planetaryscale feedback. Nevertheless, at each lag, the planetary wave feedback is large and negative in ERA-Interim. Feldstein and Lee (1998) and LH01 found a similar result. They each found that low-frequency eddies (which are of larger horizontal scale) provide a negative feedback while high-frequency eddies (which are of smaller horizontal scale) provide a positive feedback. The eddy forcing regression in Fig. 5c reveals a difference in this eddy forcing of the SAM by planetary waves between the model and reanalysis that is greater than $0.1 \mathrm{~m} \mathrm{~s}^{-1}$ day $^{-1}$ from lags of around +7 to +17 days. This can explain the difference in the decay rates of SAM anomalies in Fig. 2b. The quantification of the eddy feedback strength by $k=1-3$ waves in Figs. 5d,g (middle) reveals a dramatic difference. The negative feedback by $k=1-3$ is extremely weak in the model whereas in ERA-Interim it is almost of comparable magnitude to the positive feedback by synoptic-scale (and smaller scale) eddies. Furthermore, the planetary wave feedback is not improved in the model with bias correction. Note that this strong negative feedback by planetary-scale waves in the reanalysis is also robust to dividing that dataset in two.

When considering all zonal scales together [Figs. $5 \mathrm{e}-\mathrm{g}$ (right)], in ERA-Interim there is a large cancellation between the positive feedback by the smaller-scale waves and the negative feedback by planetary-scale waves, resulting in only a very weak positive feedback overall in the DJF season. In contrast, in the model, since the planetary wave feedback is much weaker, the positive feedback by synoptic-scale and smaller-scale eddies 
(a) $m(k>3)$ regressed onto $P C$

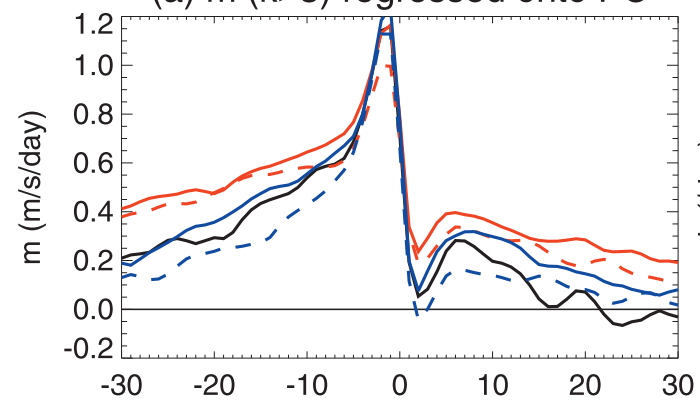

(c) $m(k=1-3)$ regressed onto PC

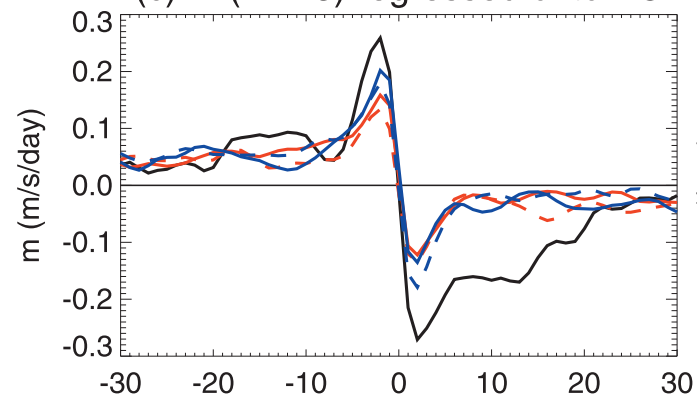

(e) $\mathrm{m}$ (all k) regressed onto $\mathrm{PC}$

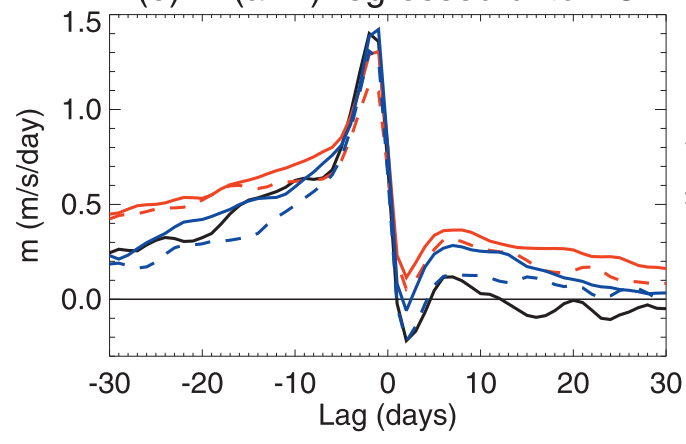

ERA

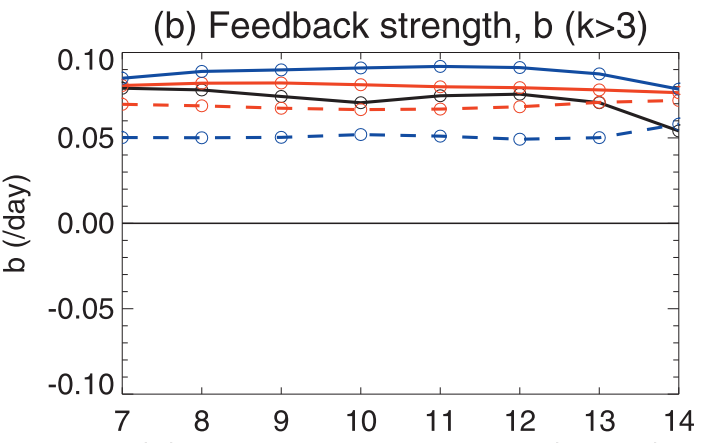

(d) Feedback strength, $b(k=1-3)$

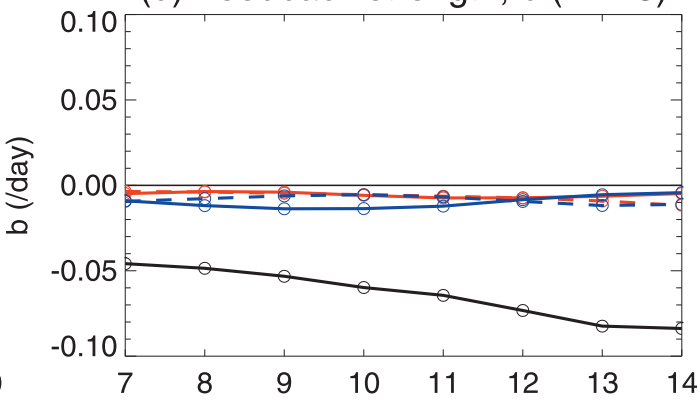

(f) Feedback strength, b (all k)

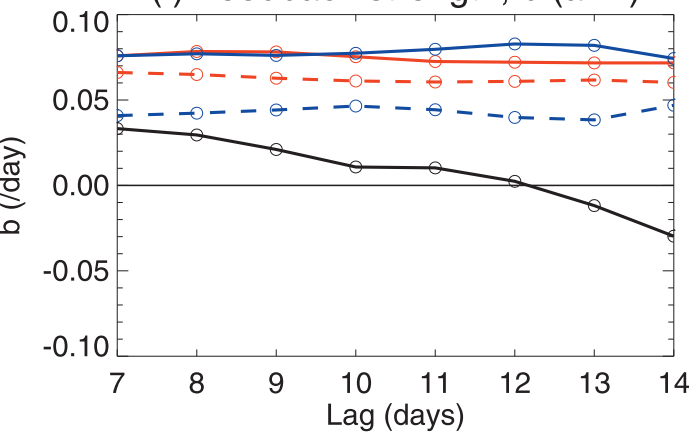

NUDG - - - - BCNUDG

(g) Average Feedback strength

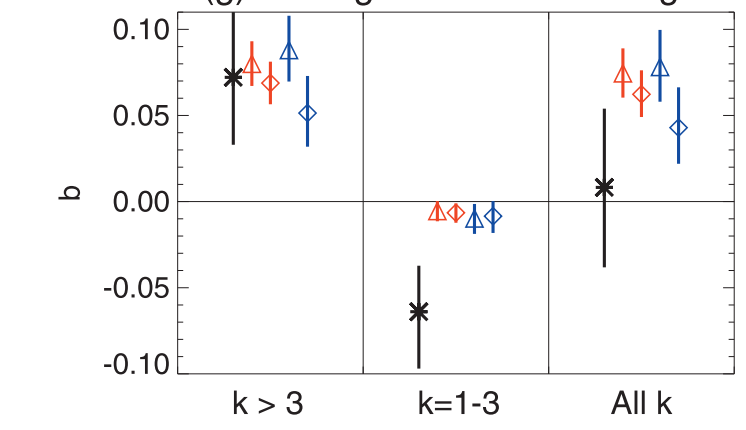

* ERA $\triangle$ FREE $\diamond B C \triangle N U D G \diamond$ BCNUDG

FIG. 5. (a)-(f) Shown are (left) lagged regression of the eddy forcing $m$ onto the SAM index and (right) feedback strength $b$ for lags 7-14 days for (top) synoptic scales and smaller, (second row) planetary scales, and (third row) all scales. (g) Here the average feedback strengths are quantified by averaging over the lags in (b), (d), and (f). The $95 \%$ confidence intervals on these mean estimates are calculated via the method outlined in the appendix. All panels are for the DJF season. 
dominates by far giving an overall strong positive feedback onto the SAM, and enhancing the SAM anomaly persistence. Note that the $b$ 's in Figs. 5b,d add up to those in Fig. 5f. A further decomposition of the planetary wave momentum flux convergence regression in Fig. $5 c$ into the wavenumber- $1,-2$, and -3 components in Fig. 6 demonstrates that most of the planetary wave feedback present in ERA-Interim and absent in the model in this season comes from the wavenumber-3 component.

So, in ERA-Interim there is a strong negative feedback by planetary-scale waves, in particular $k=3$, in the DJF season and the difference in CMAM from the reanalysis in the decay of SAM zonal wind anomalies at large positive lags in this season can be explained by the absence of this strong negative planetary wave feedback. This DJF bias will now be put in the context of the seasonal cycle of tropospheric eddy feedbacks on the SAM.

\section{b. The seasonal cycle of eddy feedbacks}

The model bias in SAM time scales has a pronounced seasonal cycle with the bias being much greater in the summer season (see Fig. 9 of Part I). To determine the seasonal cycle in SAM eddy feedbacks, the feedback strength $b$ is calculated in the same way as in the previous section using data from 90-day segments throughout the year centered at 10-day intervals. The mean feedback at positive lags of 7-14 days is quantified for each 90-day segment and plotted as a function of season in Fig. 7 with $95 \%$ confidence intervals calculated using the method outlined in the appendix. For clarity, the values for the bias-corrected and non-bias-corrected simulations are plotted separately.

At synoptic and smaller scales (Figs. 7a,b), the seasonal cycle in the eddy feedbacks is not that pronounced and, throughout the summer season, the reanalysis and model synoptic-scale feedback strengths compare well, confirming that biases in the synoptic-scale feedbacks do not contribute significantly to the bias in the summer season. There is a tendency for the model to have a positive synoptic-scale feedback that is enhanced in the winter and weaker in the summer, while the reanalysis suggests the opposite seasonality. However, the differences are within the uncertainties and in any case are in the wrong sense to provide an explanation of the summertime SAM time scale bias in the model.

In contrast, the feedback by planetary-scale waves exhibits a much more pronounced seasonal cycle. At all times of the year, both the model and ERA-Interim exhibit a negative feedback by planetary waves. In ERA-Interim, the negative feedback has a minimum in the winter season and then increases in the summer to

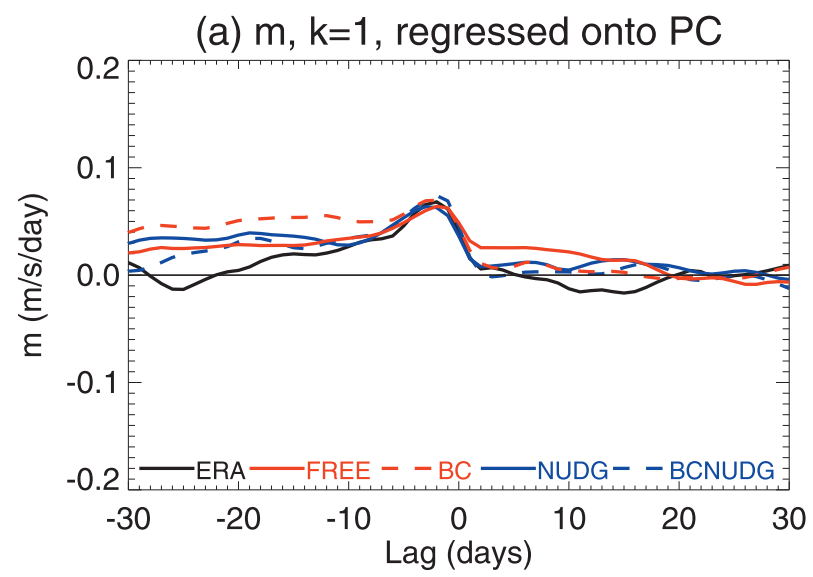

(b) $\mathrm{m}, \mathrm{k}=2$, regressed onto $\mathrm{PC}$

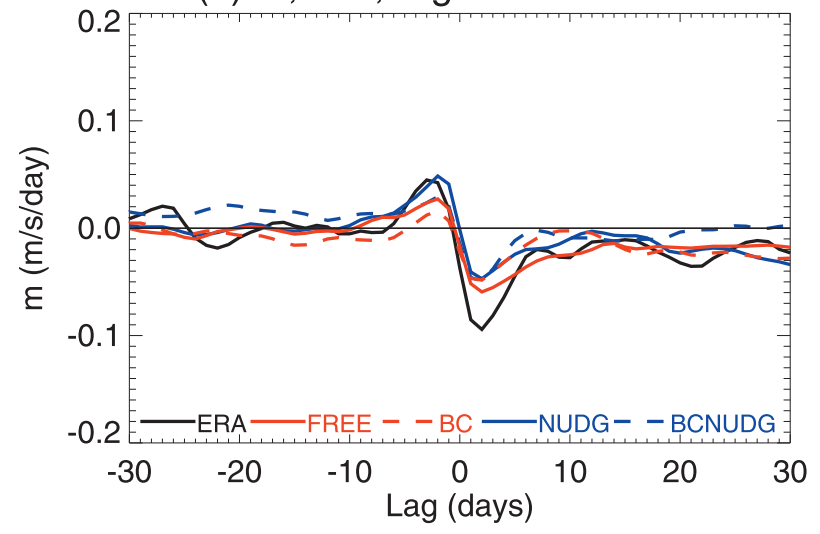

(c) $\mathrm{m}, \mathrm{k}=3$, regressed onto $\mathrm{PC}$

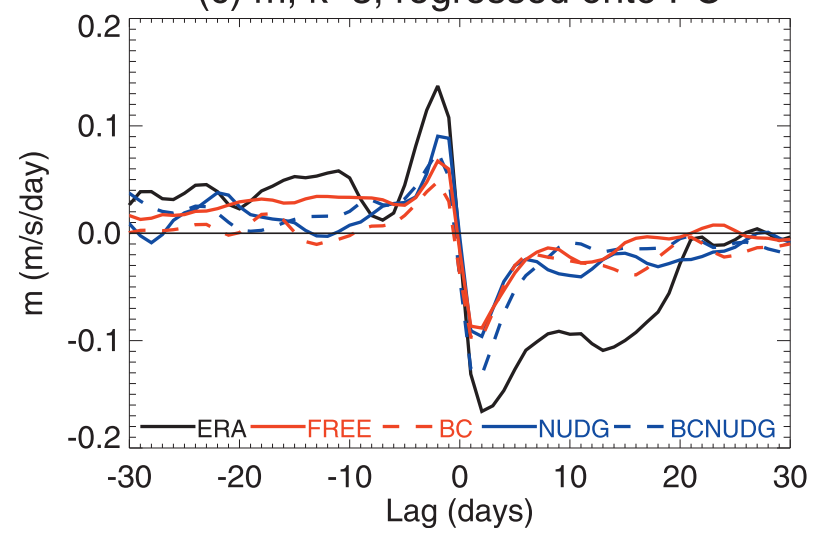

FIG. 6. Regression of the eddy forcing of the SAM $m$ onto the SAM index in DJF for zonal wavenumbers (a) $k=1$, (b) $k=2$, and (c) $k=3$.

a maximum in March. In the model, on the other hand, each simulation exhibits the largest negative feedback in the winter months and there is a minimum in the strength of this negative feedback in the summer season. For the months of November-March the negative feedback by planetary-scale waves is significantly weaker in the model than in the reanalysis, which helps explain why the bias in SAM time scales is so large in this season. 
(a) $k>3$, FREE and NUDG

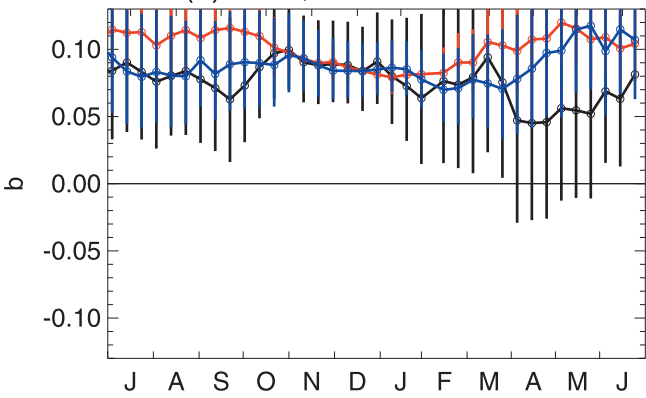

(c) $k=1-3$, FREE and NUDG

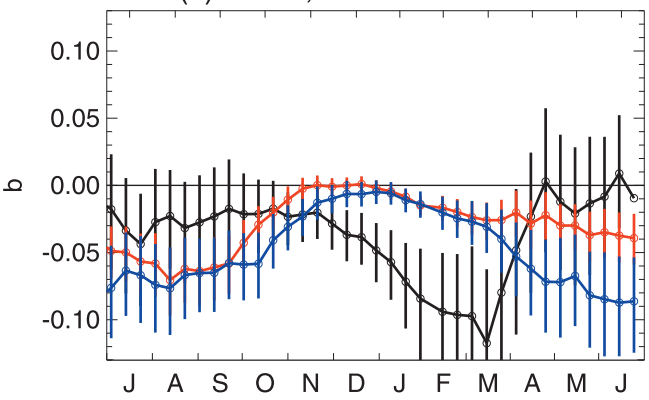

(e) All k, FREE and NUDG

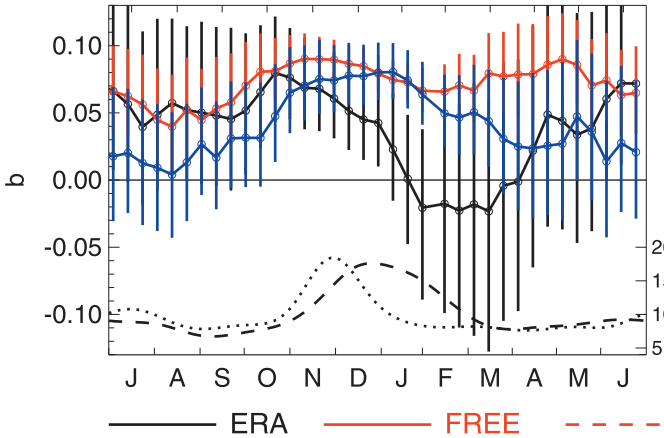

(b) $\mathrm{k}>3, \mathrm{BC}$ and BCNUDG

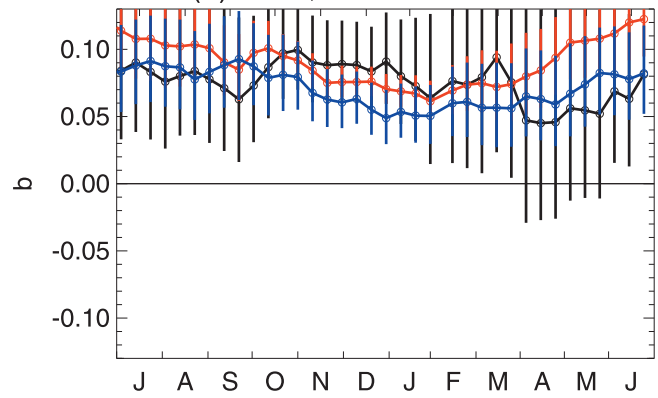

(d) $k=1-3, B C$ and BCNUDG

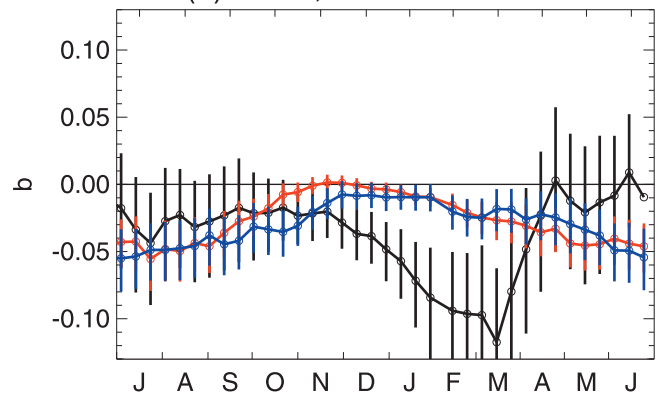

(f) All k, BC and BCNUDG

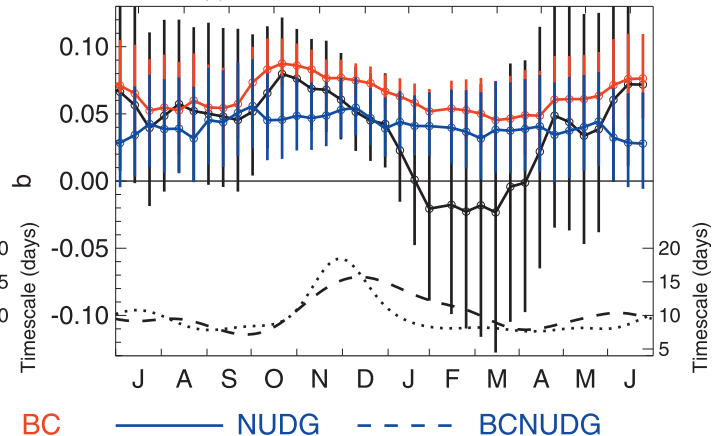

FIG. 7. Feedback strength $b$ as a function of season for (a),(b) synoptic and smaller scales $(k>3)$, (c),(d) planetary scales $(k=1-3)$, and (e),(f) all zonal scales together. Each point represents the feedback calculated from a 90-day segment centered on that date. The $95 \%$ confidence intervals are calculated by the method outlined in the appendix. The dashed lines in (e) and (f) are the SAM time scales for NUDG and BCNUDG, respectively, and the dotted line is the SAM time scale for ERA-Interim.

If the SAM persistence is completely determined by the strength of the eddy feedbacks then its seasonal variation should be related to the seasonal variation in the strength of the eddy feedbacks. However, as discussed in the appendix, longer time scale forcing of the SAM may substantially alter the SAM persistence, making it difficult to relate the seasonality of SAM persistence directly to feedback strength in the reanalysis and in our simulations with stratospheric variability. In NUDG and BCNUDG, where stratospheric variability is absent and SSTs are climatological, we may expect more of a correspondence. This is the case, broadly speaking, as seen in Figs. 7e,f. The eddy feedback tends to be greater in the summer, as are the SAM time scales. But, the correspondence is certainly not perfect and it almost appears that the seasonal cycle in SAM persistence bears more resemblance to the planetary-scale feedback strength.

There are, however, several caveats that may make it difficult to find a direct relationship between SAM persistence and feedback strength over the seasonal cycle. The relatively rapid changes in the circulation during the transition season may act to disrupt SAM anomalies, resulting in a shorter time scale, and it is also possible that surface friction exhibits some seasonal variation. Another factor to consider is that we have assumed a simple linear model of the feedback; while this works well enough for us to quantify the feedback differences between the model and reanalysis, it is by no means exact. This may account for the small differences 
in feedback strength in the simulations with and without stratospheric variability, which should have the same tropospheric dynamics; since the magnitude of the annular mode anomalies differ, any nonlinearity in the true feedback could lead to a difference in the quantified feedback strength. Another issue may be that our method of feedback strength calculation depends on the regression structures at lags of 7-14 days. This works well in the summer season when the time scales are long, but in other seasons where the time scales are shorter the regression structures at these lags may be more prone to sampling errors, as indicated by the larger uncertainties in other seasons in Fig. 7.

The important point to be taken from Fig. 7 is that, in the season where a bias in internal tropospheric dynamics has been identified, through a positive bias in SAM time scales in NUDG and BCNUDG (see Figs. 7e,f), there is a clear difference in the eddy feedback strength between ERA-Interim and the model, which is associated with the planetary scales (Figs. 7c,d). The difference in feedback strength is sufficiently large and corresponds sufficiently well with the seasonality of the model bias that we can be confident that it is indeed significant and making a substantial contribution to the model bias.

Overall, the seasonal evolution of the eddy feedback strengths in Figs. 7e,f reveals that the observed atmosphere exhibits a pronounced seasonal cycle with weaker feedbacks from December to March. This can be attributed to an enhancement of the negative feedback by planetary-scale waves during that season (Figs. 7c,d). In the model, the seasonal cycle in planetary wave feedbacks differs and actually exhibits a minimum in the summer months. This is true regardless of whether the climatological wind biases are corrected (Fig. 7d) or not (Fig. 7c). Simpson et al. (2011) demonstrated that stratospheric variability results in a substantially increased persistence in the springtime/early summer in the model. We hypothesize that a similar enhancement occurs in association with stratospheric variability in the reanalysis but is then tempered by the weakened positive eddy feedbacks later in the summer. In the model, the eddy feedbacks do not weaken and the enhanced persistence that occurs around the timing of the stratospheric vortex breakdown extends much later into the summer season.

\section{The summertime planetary wave feedback bias}

Having demonstrated an important role for planetary wave feedbacks in SAM variability in the reanalysis and in the difference between CMAM and the reanalysis, we now investigate this planetary wave feedback further. It is reasonable to expect the synoptic- and planetary-scale eddies to differ in their behavior in response to the SAM since they are forced by different mechanisms and thus respond differently to the zonal flow anomalies. Synopticscale eddies are generated by baroclinic instability in the region of maximum vertical wind shear (maximum baroclinicity), which coincides with the latitude of the midlatitude jet maximum. They therefore grow in the jet region and can only propagate out of it, not into it, transferring momentum back into the jet region as they go. Therefore, synoptic-scale eddies typically tend to maintain the barotropic component of zonal flow anomalies. As the jet shifts in latitude, so too does the region of maximum baroclinicity and the source region of synoptic-scale eddies. The propagation of these eddies away from their source region then maintains the anomalous zonal flow. This has been shown to be important for the positive synoptic-scale feedback onto the annular modes by Robinson $(1996,2000)$ and Kidston et al. (2010).

Planetary-scale waves, on the other hand, are generally produced by different processes such as flow over topography, land-sea temperature contrasts, or tropical convection and therefore can be produced at locations that are external to the tropospheric jet. They can then propagate into the jet, carrying their negative pseudomomentum as they go, thereby decelerating the zonal flow where they dissipate. Indeed, this oppositely signed influence of synoptic- and planetary-scale waves on the zonal mean flow has been found in various different studies (e.g., Shepherd 1987b; Robinson 1991).

The details of the zonal mean planetary-scale eddy feedbacks for ERA-Interim, FREE, and BC are examined in Fig. 8 (NUDG and BCNUDG exhibit similar behavior to FREE and BC). The anomalous quantities are regressed onto the SAM, averaged over positive lags of 7-14 days and defined such that they represent anomalies that occur with a poleward shifting of the jet. In the top row, the zonal wind anomalies are shown. These are larger in the model simulations since the SAM anomalies decay more slowly. The second and third rows show the poleward momentum flux anomalies for $k=1-3$ and $k=3$ respectively. The planetary-scale poleward momentum flux anomalies in ERA-Interim (Fig. 8b) exhibit a strong, relatively barotropic, decrease centered around $55^{\circ} \mathrm{S}$. The result of this is a reduced convergence of momentum to the $60^{\circ} \mathrm{S}$ region and an enhanced convergence of momentum to lower latitudes (i.e., a negative feedback on the zonal wind anomalies in Fig. 8a). In contrast, the decrease in poleward momentum flux around $50^{\circ} \mathrm{S}$ is much weaker in both the free-running and bias-corrected simulations (Figs. 8f,j), indicating the much weaker negative feedback by 
(a) u, ERA

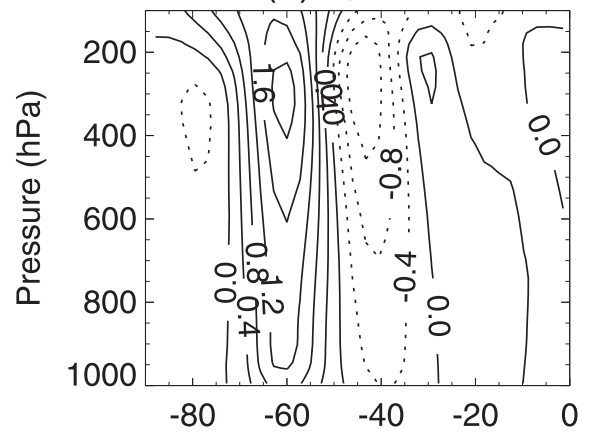

(b) - $u^{\prime} v^{\prime}, k=1-3$, ERA

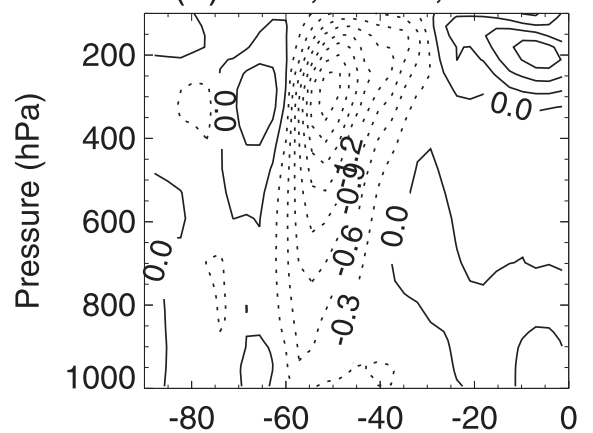

(c) $-u^{\prime} v^{\prime}, k=3$ ERA

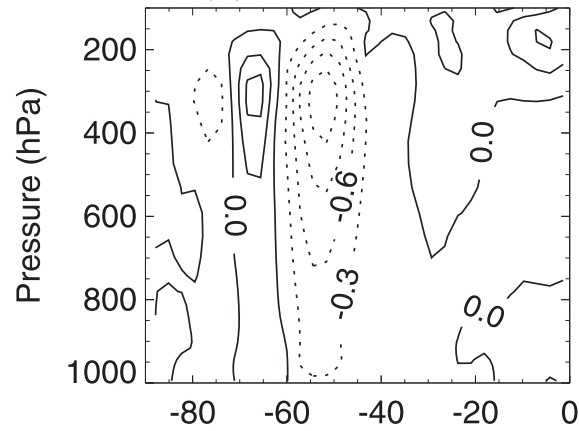

(d) E-P Flux, $k=3$, ERA

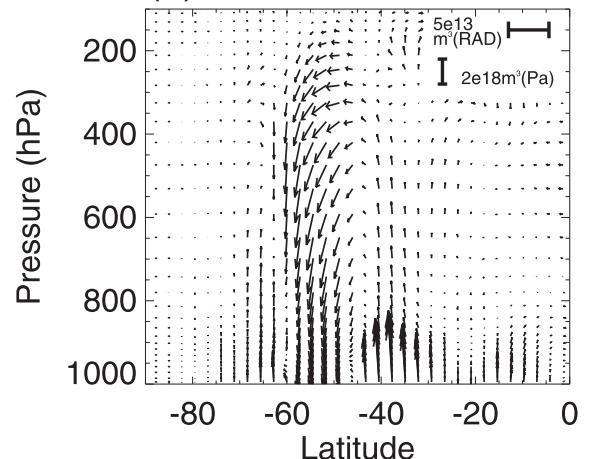

(e) u, FREE

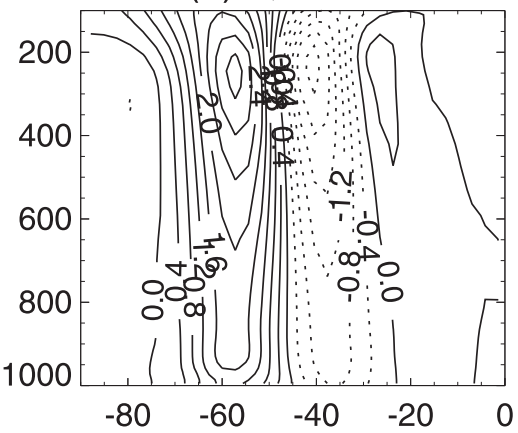

(f) $-u^{\prime} v^{\prime}, k=1-3$, FREE

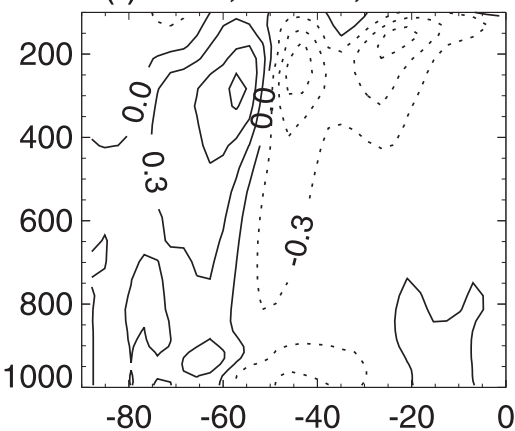

(g) $-u^{\prime} v^{\prime}, k=3$, FREE

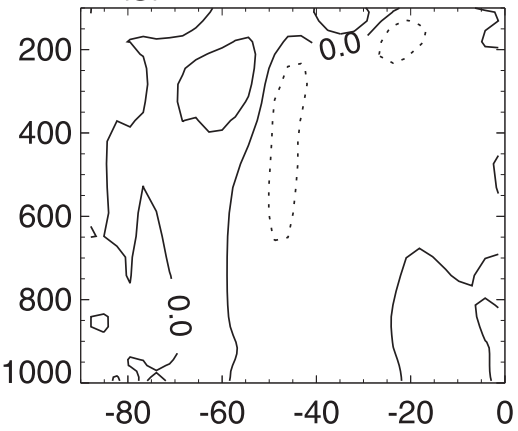

(h) E-P Flux, k=3 FREE

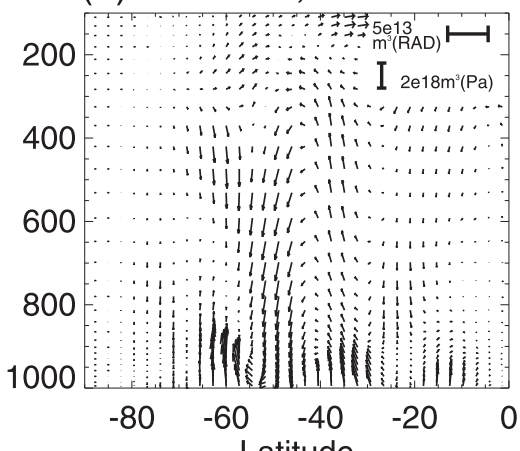

(i) $\mathrm{u}, \mathrm{BC}$

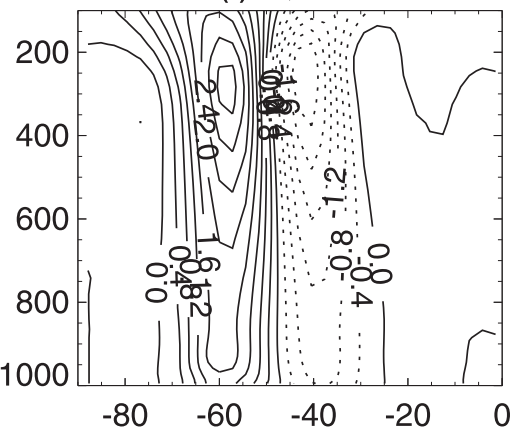

(j) $-u^{\prime} v^{\prime}, k=1-3, B C$

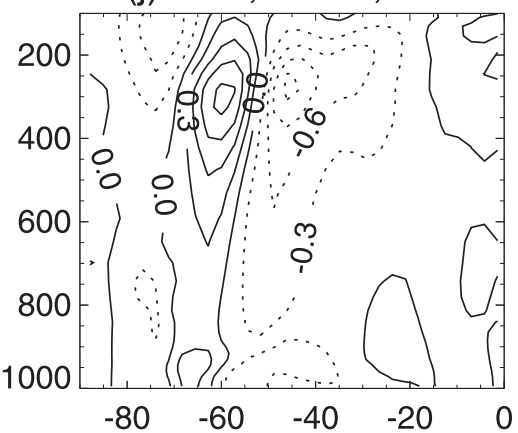

(k) - $u^{\prime} v^{\prime}, k=3, B C$

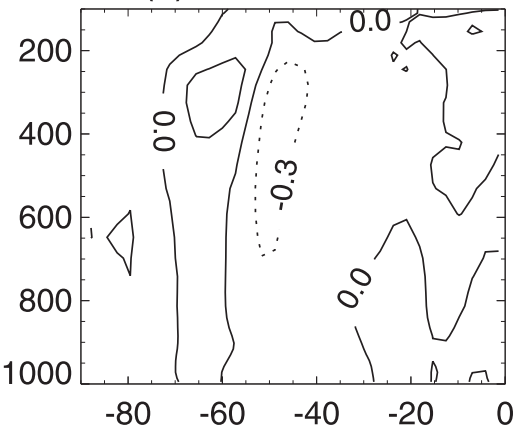

(I) E-P Flux, k=3, BC

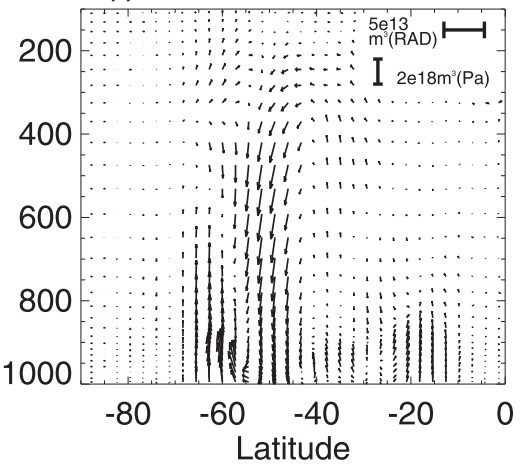

FIG. 8. The planetary wave feedback in the DJF season. Fields regressed onto the SAM index and averaged over lags +7 to +14 days are shown for (left) ERA-Interim, (center) FREE, and (right) BC. Shown are (top) zonal wind ( $\mathrm{m} \mathrm{s}^{-1}$ ), (second row) wavenumbers 1-3 poleward eddy momentum flux $\left(\mathrm{m}^{2} \mathrm{~s}^{-2}\right)$, (third row) wavenumber-3 poleward eddy momentum flux $\left(\mathrm{m}^{2} \mathrm{~s}^{-2}\right)$, and (bottom) wavenumber-3 E-P flux. Solid contours denote positive values and dashed contours denote negative values. 
planetary-scale waves in the model. Figure $8 \mathrm{c}$ again demonstrates that a significant proportion of the planetaryscale anomalies are coming from wavenumber 3 in ERA-Interim, and Figs. 8g,k demonstrate that these wavenumber-3 momentum flux anomalies are extremely weak in both model simulations. The wavenumber-3 Eliassen-Palm (E-P) flux anomalies in the bottom row of Fig. 8 demonstrate an enhanced meridional propagation of wave activity from lower latitudes (and to a lesser extent higher latitudes) toward the $60^{\circ} \mathrm{S}$ region in ERA-Interim. This is where the zonal wind anomalies maximize and this behavior is therefore consistent with the findings of LH01. They propose that SAM zonal wind anomalies alter the propagation of "external" Rossby waves through an anomalous index of refraction. As the jet shifts, the maximum in the index of refraction shifts with it. Rossby waves propagate toward this shifted maximum resulting in barotropic momentum flux anomalies. In the model, it appears, this response is much weaker.

Further insight can be gained by examination of the latitude-longitude structure of this momentum flux feedback. In Fig. 9 the zonal wind and $k=1-3$ momentum flux anomalies on the $300-\mathrm{hPa}$ level regressed onto the SAM index and averaged over positive lags of 7-14 days are shown. For ERA-Interim (Figs. 9a,d), this reveals that accompanying a fairly zonally symmetric poleward shift in the jet is a very zonally localized planetary wave poleward momentum flux anomaly. In particular, the decreased poleward $u^{\prime} v^{\prime}$ seen in the zonal mean in Fig. $8 \mathrm{~b}$ is localized to the western South Pacific, southwest of New Zealand. In FREE and BC, a localized momentum flux anomaly occurs in the same region but it is much weaker than the reanalysis, although slightly larger in $\mathrm{BC}$ compared to FREE. Therefore, it appears that, in order to understand the eddy feedback bias in CMAM that is contributing to the SAM time scale bias, it will be necessary to understand this localized planetary wave feedback to the southwest of New Zealand and determine why it is deficient in the model.

\section{CMIP5 analysis}

Having identified this deficiency in planetary wave feedbacks in CMAM it is important to determine whether the same deficiency is exhibited by other models. The CMIP5 archive allows this question to be addressed. Here, the eddy feedback strengths are quantified for the DJF season in the historical simulation for 20 models (those for which 6-hourly $u$ and $v$ were available at the time of writing).

The feedback strengths are calculated for the period 1950-2005 using the methods outlined in section 3 and the appendix. However, for CMIP5, the 6-hourly data are only available on three pressure levels: 850,500 , and $250 \mathrm{hPa}$. The pressure-weighted vertical average over the depth of the troposphere is performed from the climatological surface pressure to $100 \mathrm{hPa}$ using only these three levels (i.e., a much coarser resolution than used in the previous sections). There may be some quantitative differences between results obtained using only these three levels and those obtained with a higher vertical resolution, but our aim here is to compare the models with the reanalysis and CMAM and so this can be achieved by treating each model and the reanalysis in the same way. Therefore, in the following, the CMAM and ERA-Interim analysis has been redone using only these three vertical levels.

The eddy feedback strengths are compared with the SAM time scale that is obtained here using the daily mean zonal mean zonal wind for each model. The method of time scale calculation is the same as that used in Part I and other studies (e.g., Gerber et al. 2010) but daily mean zonal wind rather than geopotential height is used as it is available for more models. The autocorrelation function of the principal component time series $\mathrm{PC}(t)$ of the first EOF of vertically averaged (using the 850-, 500-, and $250-\mathrm{hPa}$ levels) zonal wind is obtained as a function of day of the year and lag. This is then smoothed over a 181-day window using a Gaussian filter with a full width at half maximum of around 42 days (standard deviation $\sigma=18$ days). The $e$-folding time scale $\tau$ of this smoothed autocorrelation function is then calculated using a least squares fit to an exponential up to a lag of 50 days. The average time scale over the DJF season is then obtained.

Figure 10 shows the DJF time scale versus eddy feedback strength divided into (a) $k=1-3$, (b) $k>3$, and (c) all $k$ for each of the CMIP5 models. ERA-Interim and the FREE CMAM simulation are also shown for comparison. A first point to note is that comparison of the ERA-Interim and FREE feedback strengths with those in Fig. $5 \mathrm{~g}$ shows some minor quantitative differences associated with the coarser vertical resolution used, particularly for the synoptic scales, but these are small and the same conclusions can be drawn as with the full vertical resolution.

Figure 10a shows that all models exhibit a planetaryscale feedback that is too weak compared to ERAInterim. The majority of the models are clustered just on the negative side of zero, much like CMAM. Furthermore, the DJF SAM time scale is highly correlated with the strength of the planetary wave feedback (correlation = 0.82). A few models do appear to do better at exhibiting this negative planetary wave feedback, in particular the intermediate resolution Norwegian Earth System 
(a) SAM u, ERA

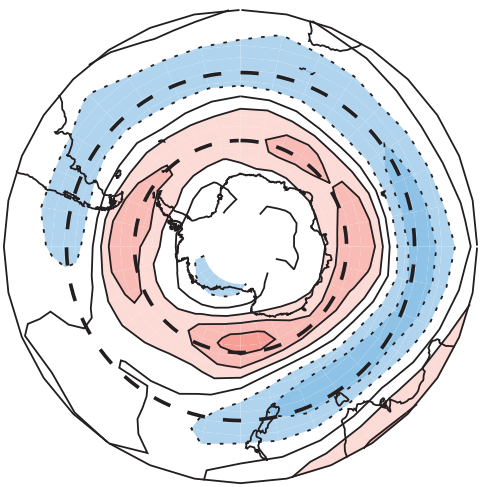

(d) SAM - $u^{\prime} v^{\prime}, k=1-3$, ERA

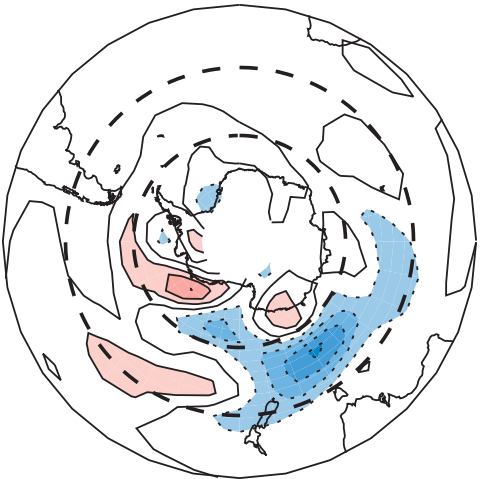

(g) $C \lim -\left(u^{\prime} v^{\prime}\right)^{\prime}$, ERA, $k=1-3$

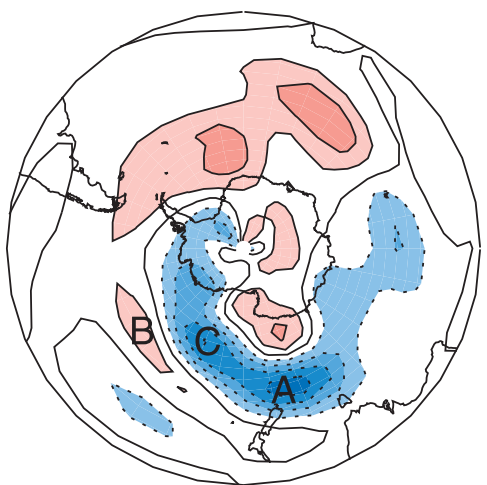

(b) SAM u, FREE

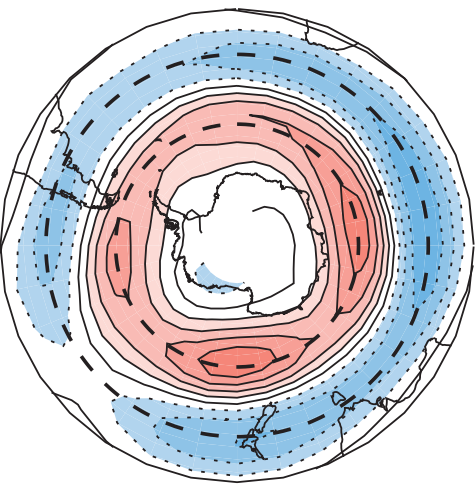

(e) SAM - u'v', $k=1-3$, FREE

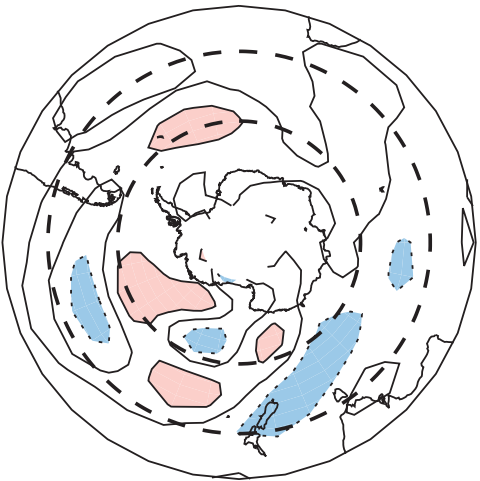

(h) Clim -(u'v')', FREE, $k=1-3$

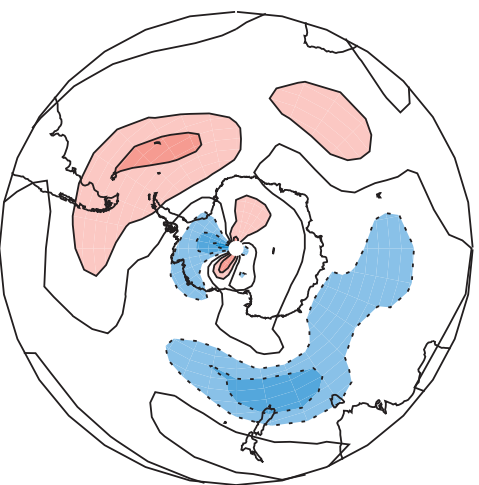

(c) SAM u, BC

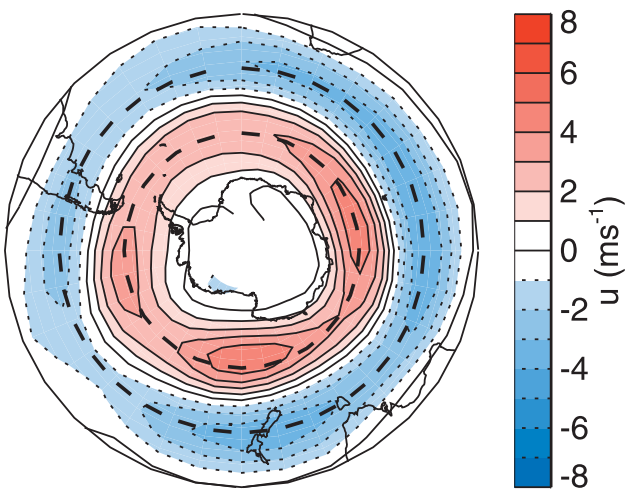

(f) SAM - $u^{\prime} v^{\prime}, k=1-3, B C$

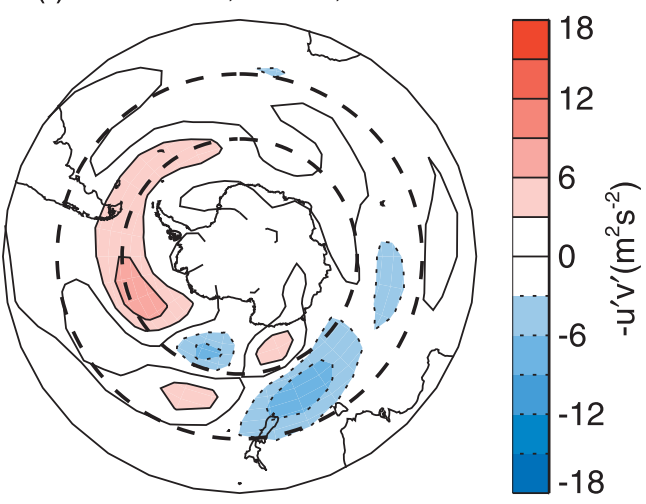

(i) $\mathrm{Clim}-\left(u^{\prime} v^{\prime}\right)^{\prime}, \mathrm{BC}, \mathrm{k}=1-3$

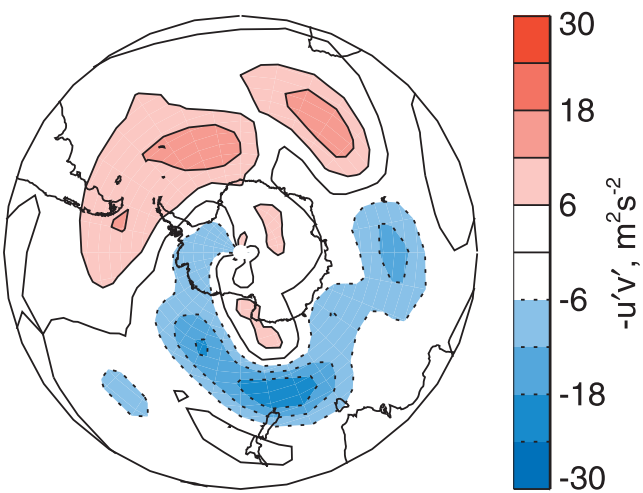

FIG. 9. Lagged regression of lat-lon fields on the 300-hPa level onto the SAM index averaged over positive lags from 7-14 days, for (top) zonal wind and (middle) wavenumbers 1-3 poleward eddy momentum flux. (bottom) Climatological 300-hPa wavenumbers 1-3 poleward eddy momentum flux. For all rows, data are for (left) ERA-Interim, (center) FREE, and (right) BC. In (bottom), the anomaly from the zonal mean is shown to highlight zonal asymmetries and both stationary and transient components are included. Note that even without subtracting the zonal mean, the poleward eddy momentum flux is negative climatologically around point A, indicating poleward propagation of planetary wave activity in this region. The dashed lines in (top) and (middle) indicate the latitude of the maximum and minimum zonal mean zonal wind anomalies in each case.

Model, version 1 (NorESM1-M), and Community Climate System Model, version 4 (CCSM4). However, NorESM1-M does not seem to be getting it right for the right reason since its planetary wave feedback is not localized in the southwest Pacific (not shown). CCSM4 does seem to do relatively well in this regard. The spatial structure of the eddy feedback for each of the other models (not shown) demonstrates that they are all 
(a) DJF timescale vs $k=1-3$ feedback strength

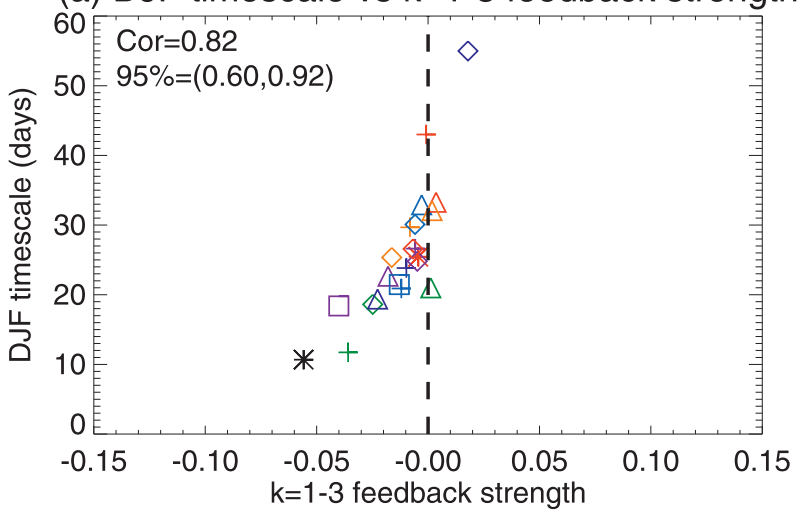

(b) DJF timescale vs $k>3$ feedback strength

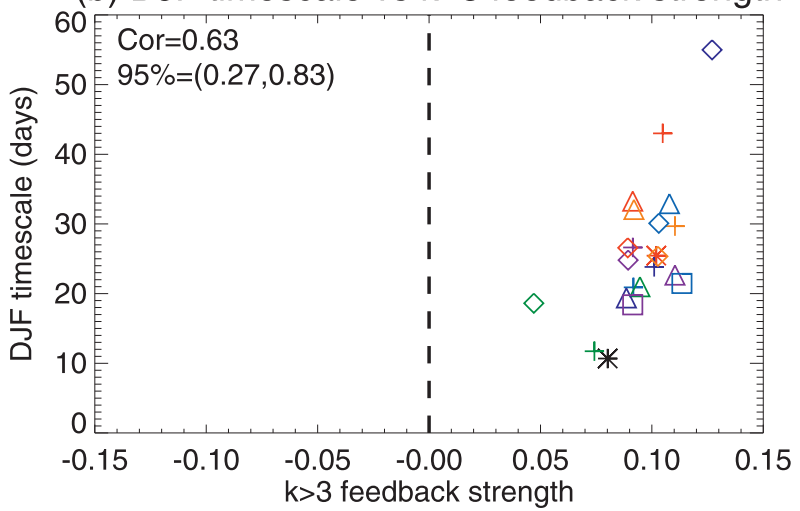

(c) DJF timescale vs all $\mathrm{k}$ feedback strength

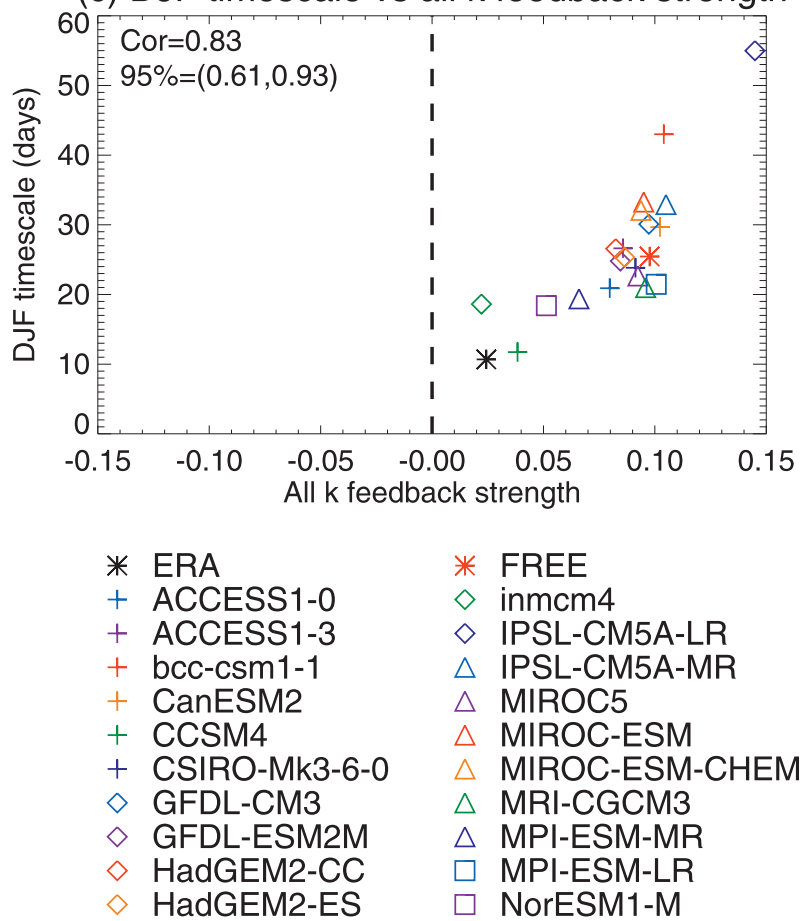

FIG. 10. DJF averaged SAM time scale vs feedback strength for (a) $k=1-3$, (b) $k>3$, and (c) all zonal wavenumbers. The CMIP5 models, ERA-Interim, and the FREE CMAM simulation are lacking in the negative feedback localized to the southwest of New Zealand, in a similar manner to CMAM.

Figure 10b then compares the SAM time scale with the $k>3$ feedback strength. Most models exhibit a synoptic-scale feedback that is stronger than that of the reanalysis but this bias is much smaller than the bias in the planetary-scale feedbacks. Again, the DJF time scale is correlated with the $k>3$ feedback strength (correlation $=0.63$ ) although this correlation is much weaker than for the planetary-scale waves.

When all scales are considered together in Fig. 10c, the overall eddy feedback strength is much stronger in the majority of models than it is in the reanalysis and the overall feedback strength is highly correlated with the SAM time scale (correlation $=0.83$ ). The stronger eddy feedbacks in the models will contribute to the overly persistent SAM and therefore likely explain at least some fraction of the time scale bias. Furthermore, in the majority of models a significant proportion of this bias in eddy feedback strength is arising from a deficiency in the negative feedback by planetary-scale waves, which in the reanalysis has been shown to be primarily associated with wavenumber 3 . So, this deficiency in planetary wave feedbacks that has been identified in CMAM is exhibited by virtually all state-of-the-art GCMs.

\section{Discussion}

\section{a. Wave propagation and the planetary wave feedbacks}

The reanalysis exhibits a strong negative planetary wave feedback onto the SAM that is localized to the region southwest of New Zealand. This localized momentum flux anomaly in response to the SAM in Fig. 9d can be understood through ray tracing arguments if there are external Rossby waves propagating toward the jet from lower latitudes in that region. Consider a Rossby wave propagating into the jet from lower latitudes, depicted schematically in Fig. 11. This schematic depicts the expected tilt of phase lines and the propagation of wave activity of such a wave following the ray tracing arguments of Shepherd (1987a, see his Fig. 3). As with refractive index arguments, ray tracing arguments assume a scale separation between the waves and the mean flow that is generally not well respected in the

shown. The correlation between the SAM time scale and feedback strength is noted in the top left corner of each panel along with the $95 \%$ confidence interval on the correlation coefficient calculated using the Fisher transform method (Devore 1999). 

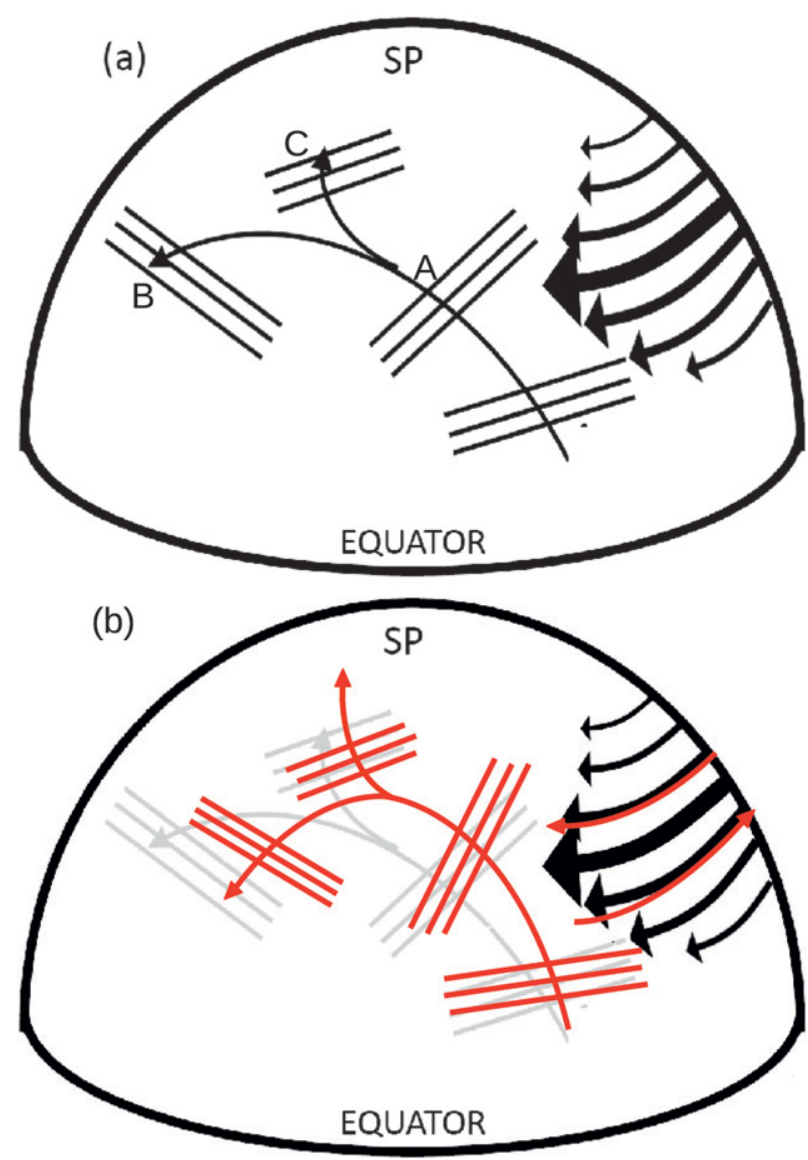

FIG. 11. (a) Schematic depiction of the behavior of a Rossby wave propagating into the shear of the midlatitude jet from lower latitudes, following the ray tracing arguments of Shepherd (1987a). Relate the locations marked A, B, and C to those in Fig. 9g. (b) Schematic depiction of the change in wave propagation associated with an enhanced meridional wind shear across the jet center (i.e., a positive SAM state). The climatological wave behavior is depicted in gray, and the behavior under positive SAM conditions is depicted in red.

atmosphere. However, these ray tracing ideas do seem to work well to explain the observed momentum fluxes. Poleward propagating waves in the $\mathrm{SH}$ have their phase lines leaning from southwest to northeast. As the waves propagate through the westerly shear the phase lines are tilted toward alignment with meridians, decreasing their meridional wavenumber (point A in Fig. 11a). The wave may then reach a point where the meridional wavenumber passes through zero (corresponding to a minimum in the refractive index or a turning latitude) upon which the phase lines begin to tilt away from meridians in the opposite sense (southeast to northwest) and the wave activity propagates equatorward (point B). The other possibility is that the waves do not reach a turning latitude before they pass through the jet maximum and continue propagating poleward into a region of oppositely signed shear upon which the tilting of phase lines will be reversed (point C). During poleward propagation the meridional tilt of the eddies is associated with an equatorward momentum flux and vice versa. Climatologically the planetary wave momentum fluxes exhibit a structure that resembles this behavior (cf. points A, B, and C in Fig. 9g with those in Fig. 11a). This suggests that climatologically there is propagation of wave activity toward the jet in the region west of New Zealand following these ray tracing arguments, resulting in a localized equatorward momentum flux to the southwest of New Zealand.

In the case of a positive SAM state (depicted schematically in Fig. 11b) the jet maximum has shifted poleward. This allows the waves to propagate farther meridionally until they reach their turning point, which is now located at a higher latitude. The result is an enhanced equatorward momentum flux between the negative and positive zonal flow anomalies, as is seen in Fig. 9d. The pattern in Fig. 9d looks to be a poleward extension of the climatological momentum flux in response to the poleward shifting of the jet in a similar manner to Fig. $11 b$.

This discussion of the behavior of waves in response to the SAM is analogous to the arguments of LH01 based on refractive index, but provides a spatial perspective. The negative feedback that is localized to the southwest of New Zealand (Fig. 9d) occurs in a region where climatologically there is poleward propagation of wave activity and it can be explained through propagation of the waves farther poleward in response to the poleward shifting of the jet in the case of a positive SAM state.

\section{b. The relation to climatological model biases}

The bias in planetary wave feedbacks is not alleviated in the bias-corrected runs. Since bias correction improves the full 3D climatological circulation, we can conclude that climatological circulation biases are not the cause of the problem. But, it may be that climatological biases and the eddy feedback biases are related (i.e., they could have a common cause).

It is immediately apparent in Fig. 9h that the climatological planetary wave momentum fluxes to the southwest of New Zealand are much weaker in the FREE CMAM simulation than in the reanalysis, suggesting that the model is not exhibiting the correct planetary wave activity in this region. It is also clear that there are climatological stationary wave biases in this region in Fig. 12b, which shows the difference between FREE and ERA-Interim for climatological 300-hPa eddy geopotential height in the DJF season. The localized anticyclonic circulation to the south of New Zealand is too 
(a) Eddy GPH, ERA-Interim

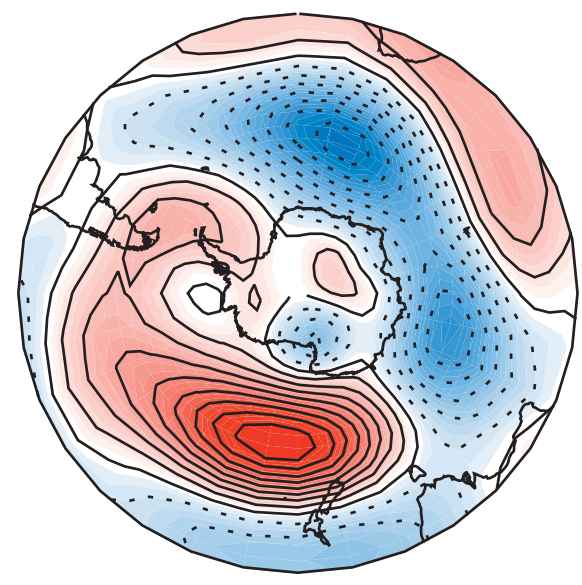

(b) FREE - ERA

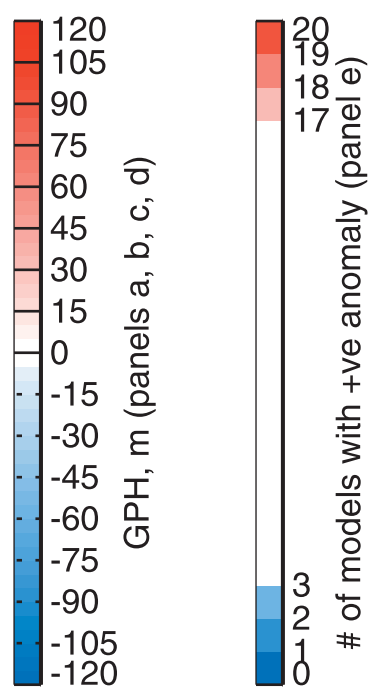

(c) BC - ERA

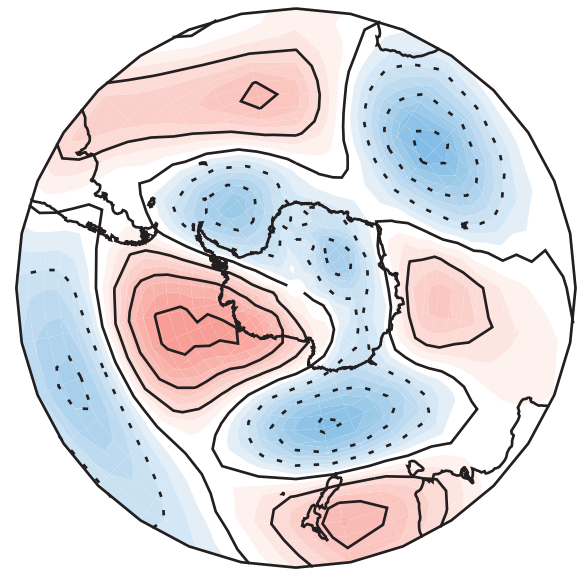

(d) CMIP-5 - ERA
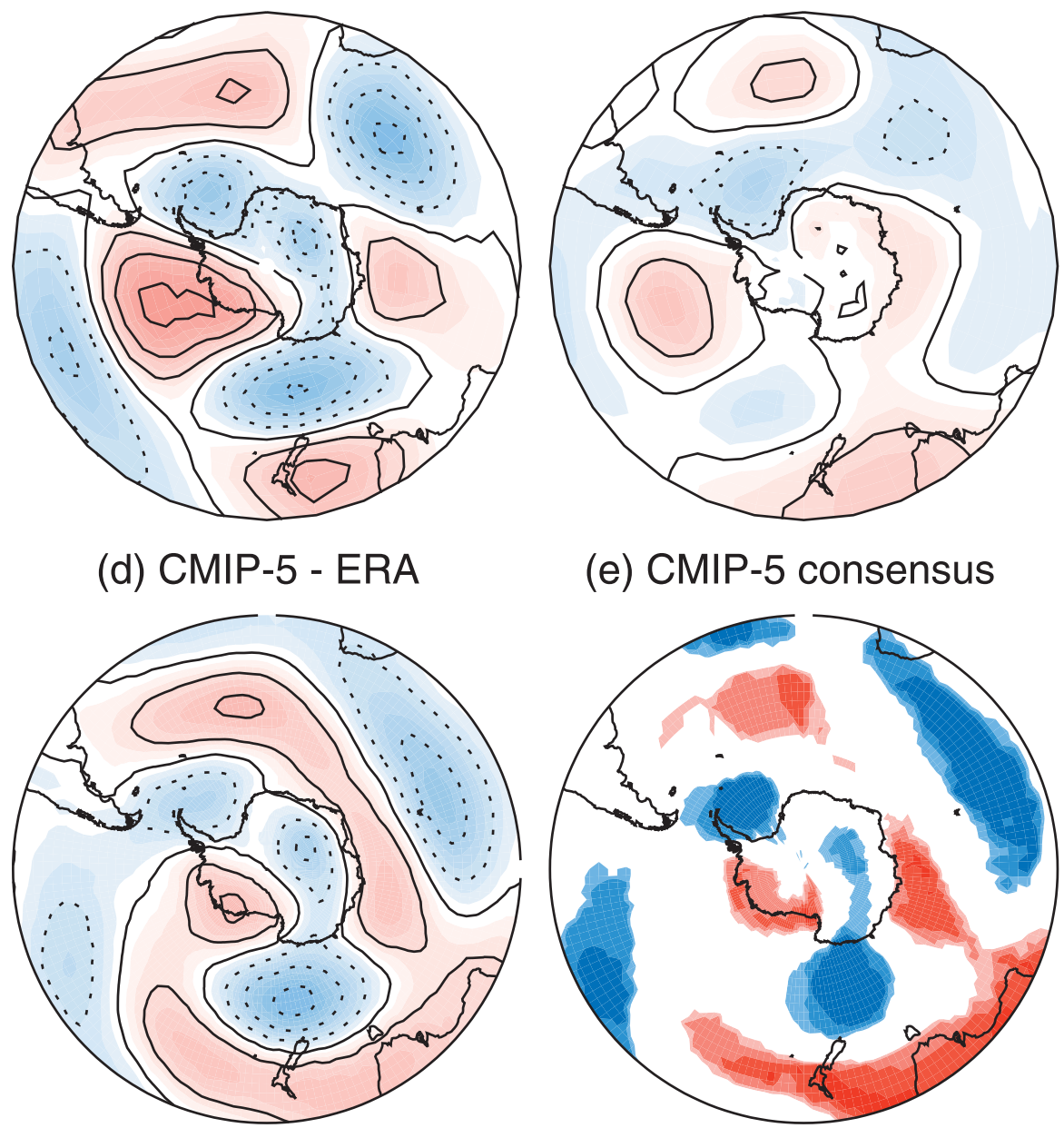

(e) CMIP-5 consensus

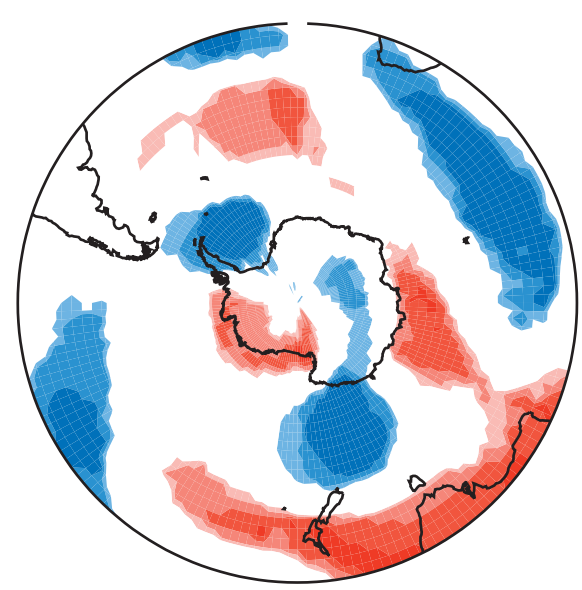

FIG. 12. DJF climatological 300-hPa geopotential height (anomaly from the zonal mean) for (a) ERA-Interim, (b) FREE - ERA-Interim, (c) BC - ERA-Interim, (d) the CMIP5 multimodel mean - ERA-Interim, and (e) the consensus among the CMIP5 models on the sign of the geopotential height bias relative to ERA-Interim. The white regions in (e) indicate where less than $85 \%$ of the models agree on the sign, blue indicates where more than $85 \%$ agree on a negative bias, and red indicates where more than $85 \%$ agree on a positive bias. 
weak in CMAM. Furthermore, the CMIP5 multimodel mean exhibits a bias in this region that is strikingly similar to CMAM (Fig. 12d), and indeed all the CMIP5 models exhibit an anticyclonic circulation that is too weak to the south of New Zealand (Fig. 12e). However, both the climatological wave field (Fig. 12c) and the climatological momentum flux (Fig. 9i) are substantially improved in this region by the bias correction in CMAM.

If the feedback bias is related to these climatological biases, then the explanation must account for the fact that bias correcting the climatology does not improve the feedbacks. One possibility is that transience is somehow important. Bias correction improves the climatological wave field but it does not necessarily mean that the transient waves that sum up to that climatology are improved. Indeed, less than $30 \%$ of the planetary wave feedback in ERA-Interim is associated with the stationary wave component and it can be seen in Fig. 9i that, while the climatological circulation has been improved in BC, there is still some bias in $u^{\prime} v^{\prime}$. This is not too surprising since climatologically $u^{\prime} v^{\prime}$ consists of both stationary and transient components and bias correction does not guarantee an improvement in the transient component. For example, one possibility is that the models are not representing the generation of transient planetary waves from the tropical western Pacific correctly. Such waves, as they follow great circle paths, would reach the midlatitudes in this region to the southwest of New Zealand. The study of Ding et al. (2012) has identified wave structures in this region in the reanalysis that appear to be related to tropical processes and so the tropics is one location in which to search for an underlying cause of biases in planetary wave structures and feedbacks in this region.

Another possibility is that the discussion above on the altered propagation of planetary waves in response to the SAM is incomplete and that, as well as changing the eddy propagation, the SAM alters the source of Rossby waves. For example, the climatological biases in Fig. 12 may be indicative of some deficiency in Rossby wave generation around the New Zealand region. The SAM may alter the generation of Rossby waves in this region in the real atmosphere but, in the models, if this generation process is deficient then this will not happen, even in the case where we artificially improve the climatology by bias correction.

\section{c. Comparison of the seasonal cycle in eddy feedbacks with previous studies}

Aside from improving our understanding of the cause of the SAM time scale bias in CMAM, this analysis has also provided insights into certain aspects of the dynamics of SAM variability in the observed atmosphere. Figure 7 has revealed a seasonality in the strength of the zonal mean eddy feedbacks on the SAM in the reanalysis with the strength of the feedback being much reduced in the summer season from around December to March. This can be attributed to an enhancement of the negative feedback by planetary waves (in particular planetary wave 3) in the summer season, which largely offsets the positive feedback from synoptic-scale eddies. In a recent study, Barnes and Hartmann (2010) examined the dynamics of SAM variability in the summer and winter seasons. Rather than using a zonal mean framework, they examined the 3D vorticity budget and demonstrated that the winter SAM is much less zonally symmetric: the eddy-driven jet is weak in the Pacific sector and the circulation is dominated by the subtropical jet there. Consequently the dominant mode of variability in the Pacific in winter was found to be a pulsing of the jet, rather than a latitudinal shifting. They demonstrated that this pulsing does not invoke the same eddy feedbacks as a latitudinal shifting does. Based on this, they hypothesized that the SAM is more persistent in the summer season than in the winter because in the summer the overall eddy feedbacks are stronger (i.e., the opposite of what is found here). Their reasoning was that in the summer the SAM represents a zonally symmetric latitudinal shifting of the jet that invokes a positive eddy feedback, whereas in the winter the latitudinal shifting and the positive eddy feedbacks are localized to the Indian Ocean sector.

Here, we find that if the eddy feedbacks on the zonal mean SAM as a whole are considered, the total eddy feedback on SAM zonal mean zonal wind anomalies is actually stronger in the winter than in the summer. Rather than being due to differences in the dynamics of the SAM between the seasons, the present work suggests that the SAM is more persistent in the SH summer than the winter because of the presence of stratospheric variability that occurs in the late spring/early summer and acts to force persistent SAM anomalies in the early part of the summer season. This influence of stratospheric variability is confirmed by the difference between our nudged and free-running simulations (see Part I). That is not to say that the SAM dynamics identified by Barnes and Hartmann (2010) are not an important aspect of its seasonal variation, but rather we propose that it is not the governing factor when considering the SAM time scales.

The reason for this discrepancy requires further investigation since the use of different diagnostics makes a direct comparison between the two studies difficult. However, we can propose a couple of possibilities. First, Barnes and Hartmann (2010) presented lagged 
correlations to infer feedback strength and in the appendix we demonstrate that this may not be sufficient to infer differences in feedback strength between seasons where the intraseasonal forcing of the SAM may differ. Second, and perhaps more importantly, Barnes and Hartmann (2010) focused on the upper troposphere (a mass weighted layer between 150 and $300 \mathrm{hPa}$ ). The planetary wave feedback identified here, in Fig. 8b, has a large barotropic component. If only the upper troposphere is considered, the planetary wave feedback would appear relatively less important when compared with the positive synoptic-scale eddy feedback that is more localized to the upper troposphere. Furthermore, in the transient picture, it would be expected that eddy forcing in the middle/lower troposphere would induce a circulation anomaly above as the atmosphere adjusts. It is possible that the planetary wave forcing observed here in the vertical integral is actually showing up in the stretching term in the upper troposphere in Barnes and Hartmann (2010). There is some suggestion that this is the case in their Fig. 4a where a relatively large negative forcing of the SAM by the stretching term can be seen that is quite localized to the southwest of New Zealand-that is, the region where the planetary wave feedbacks identified in the present study are strongest.

\section{Conclusions}

The enhanced persistence of SAM variability in the summer season in GCMs relative to observations is a common problem. The comparison of eddy feedbacks on the SAM between the reanalysis and the CMAM experiments and the CMIP5 multimodel ensemble reveals a common model bias in the feedback by planetary waves, in particular zonal wavenumber 3 , onto the SAM in the summertime. In the SH summer season, in the reanalysis, the planetary waves provide a strong negative feedback onto the SAM in a localized region to the southwest of New Zealand. The models do not seem to capture this correctly, which likely accounts for the greater persistence of the simulated SAM in the summertime when compared with observations.

The fact that the time scale bias can be related to a deficiency in eddy feedbacks on the SAM is of concern for our ability to predict future changes in the $\mathrm{SH}$ since this bias in eddy feedbacks on the SAM will also likely act on SAM-like zonal wind anomalies produced by climate forcings such as ozone depletion or increased greenhouse gas concentrations. All else being equal, one might expect the models to produce SAM-like anomalies in response to forcings that are too large in the DJF season. But, GCMs [including CMAM; see McLandress et al. (2011)] have managed to simulate trends in the recent past in the $\mathrm{SH}$ midlatitude circulation reasonably well (Son et al. 2010). This bias in eddy feedbacks then raises this question: Are the models able to simulate recent trends for the correct reason?

However, the main outstanding question arising from this study is this: Why do the models not capture the planetary wave feedbacks in the SH summer correctly? The answer to this question will also have to explain the seasonality in planetary wave feedbacks and its biases. There are common biases among the models in the climatological circulation in the southwest Pacific in the summer season that are likely related to the eddy feedback problem, but the CMAM bias-corrected runs suggest that they are not the underlying cause of it. Those simulations demonstrate that a model can have the correctly climatology but still have the wrong eddy feedbacks, suggesting that getting the climatology correct for the correct reasons may be the key. We can put forward a couple of hypotheses; for instance, the models may not correctly capture the forcing of transient planetary waves from the tropics correctly or may not correctly simulate the generation of planetary waves in the vicinity of New Zealand. Work is ongoing to investigate each of these possibilities. Overall, the results suggest that an improvement in our understanding of the planetary wave feedback on the SAM and the reasons for its deficiency in GCMs is necessary to improve the fidelity of simulated SH midlatitude variability and change.

Acknowledgments. This work was funded by the Natural Sciences and Engineering Research Council of Canada, Environment Canada and a Lamont-Doherty Earth Observatory postdoc fellowship. Computing resources were provided by Environment Canada. We are grateful to Michael Sigmond for making his data for the BC simulation available, Slava Kharin for providing technical assistance in the setup of the bias correction, and Haibo Liu for aid in downloading/storage of CMIP5 data. We also thank John Fyfe, Charles McLandress, Richard Seager, and Bel-Helen Burgess for helpful comments/discussion and Walt Robinson, Dennis Hartmann, Ed Gerber, and an anonymous reviewer for helpful reviews.

We acknowledge the World Climate Research Programme's Working Group on Coupled Modelling, which is responsible for CMIP, and we thank the climate modeling groups for producing and making available their model output. For CMIP the U.S. Department of Energy's Program for Climate Model Diagnosis and Intercomparison provides coordinating support and led development of software infrastructure in partnership with the Global Organization for Earth System Science Portals. 


\section{APPENDIX}

\section{The Feedback Calculation and Its Uncertainties}

The method used here to calculate the feedback strength differs from that used in other studies (Lorenz and Hartmann 2001, 2003). We are motivated to use this different method by the fact that forcing on intraseasonal time scales can have an important influence on the SAM. The presence of intraseasonal forcing differs substantially between the seasons resulting from, for example, the presence of stratospheric variability in the SH spring (Simpson et al. 2011) and ENSO variability in the SH summer (L'Heureux and Thompson 2006). It also differs substantially between the model simulations with and without stratospheric variability. In the presence of intraseasonal forcing, the assumptions used in the method outlined in Lorenz and Hartmann (2001) break down. It is therefore important in our situation, where we are comparing seasons and simulations with different levels of intraseasonal forcing, that this be taken into account in the feedback strength methodology. This will be demonstrated in the following using a synthetically generated time series of SAM zonal wind anomalies $[\bar{u}]_{s}$ and eddy forcing of the SAM $m$.

We begin by generating a synthetic time series of the random component of the eddy forcing of the SAM $\tilde{m}$ using a second-order autoregressive (AR2) noise process according to

$$
\tilde{m}_{t}=0.6 \tilde{m}_{t-1}-0.3 \tilde{m}_{t-2}+\epsilon_{t}
$$

where subscript $t$ denotes day $t$ and $\epsilon$ is white noise distributed uniformly between -1 and +1 . A section of this synthetic $\tilde{m}$ time series is shown in Fig. A1a along with its autocorrelation function in Fig. A1c. The coefficients of the AR2 process were chosen to give an autocorrelation function that resembles that of the atmosphere (cf. Figs. 4 and A1c). Assuming a linear model of the feedback, the SAM zonal wind anomalies evolve according to

$$
\frac{\partial[\bar{u}]_{S}}{\partial t}=m-F[\bar{u}]_{S}, \quad m=\tilde{m}+b[\bar{u}]_{S},
$$

where $F$ represents a frictional drag coefficient and the eddy forcing is divided into the random component $\tilde{m}$ and a feedback component that is linearly related to the SAM zonal wind anomalies $b[\bar{u}]_{s}$. A synthetic time series for $[\bar{u}]_{s}$ and $m$ can therefore be obtained by integrating (A2) forward in time using the AR2 time series for $\tilde{m}$ described above. For example, the characteristics of the synthetic SAM generated with $F=0.1$ and $b=0.04$ are shown by the solid lines in Figs. A1d-f. These parameters were chosen to provide an autocorrelation function of $[\bar{u}]_{s}$ and a cross correlation between $[\bar{u}]_{s}$ and $m$ that resembles that of the real atmosphere as demonstrated by comparison of Figs. A1d,f with Figs. 2, 5.

Now consider a situation where there is some form of slowly varying intraseasonal forcing acting on the SAM denoted by $S$. The evolution of SAM zonal wind anomalies is now governed by

$$
\frac{\partial[\bar{u}]_{S}}{\partial t}=m-F[\bar{u}]_{S}+S, \quad m=\tilde{m}+b[\bar{u}]_{S} .
$$

Keeping the frictional and feedback parameters exactly the same, we now apply a small amplitude sinusoidally varying forcing to the SAM given by $S=0.15 \sin (2 \pi t)$ 200), a section of which is shown in Fig. A1b. This is not intended to mimic a particular forcing but simply to demonstrate what such a relatively small amplitude but long time scale forcing does to the SAM characteristics.

The autocorrelation of $[\bar{u}]_{S}$ and the cross correlation between $m$ and $[\bar{u}]_{s}$ in the presence of $S$ are shown by the dashed lines in Figs. A1d and A1f. The presence of this relatively small-amplitude intraseasonal forcing has substantially decreased the decay rate of the $[\bar{u}]_{s}$ autocorrelation function (Fig. A1d). The rate of change of the autocorrelation function as a function of lag in Fig. A1e demonstrates that this is primarily occurring through a difference in the rate of decay at small lags, leaving the autocorrelation of $[\bar{u}]_{s}$ to be larger out at large positive lags. This is similar to what was found in the comparison of simulations with and without stratospheric variability in Fig. 2 and results from the fact that in the presence of longer time scale forcing, the shorter time scale SAM anomalies driven by the random eddy forcing $\tilde{m}$ play a relatively less important role in the autocorrelation of $[\bar{u}]_{s}$.

Turning to the cross correlation between $m$ and $[\bar{u}]_{s}$ in Fig. A1f, it can be seen that the presence of this intraseasonal forcing substantially increases the cross correlation at positive lags. Our feedback parameter $b$ has not changed, but the presence of the intraseasonal forcing results in a slower decay of $[\bar{u}]_{s}$ and as a result the cross correlation between $[\bar{u}]_{s}$ and $m$ (which depends linearly on $[\bar{u}]_{s}$ ) necessarily is larger at positive lags. This demonstrates the danger of inferring the feedback strength from the cross correlation of $m$ and $[\bar{u}]_{s}$ alone, in the presence of intraseasonal forcings. The method outlined in Lorenz and Hartmann (2001) assumes that the SAM zonal wind anomalies obey (A2) without any form of externally forced SAM variability. If the eddy feedback $b$ were to be calculated using that method, in the case 
(a) Synthetic random eddy forcing time series ( $\widetilde{m})$

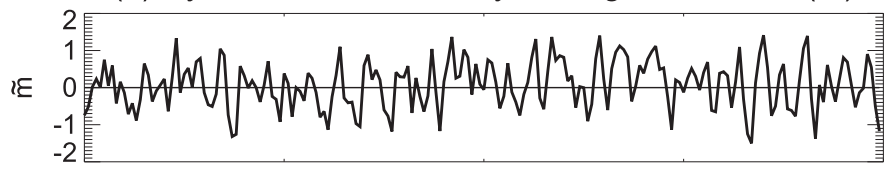

(b) Synthetic intraseasonal forcing time series (S)

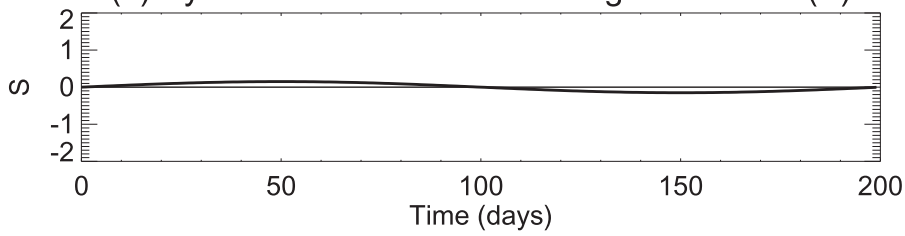

(d) $u_{s}$ autocorrelation

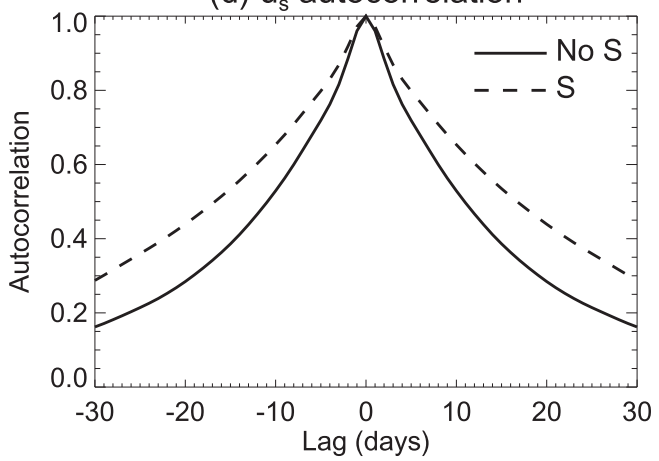

(f) $\operatorname{Cor}\left(m, u_{s}\right)$

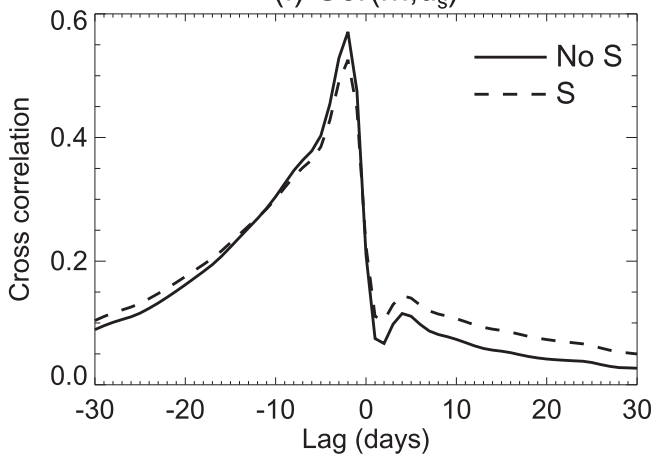

(c) $\tilde{\mathrm{m}}$ autocorrelation

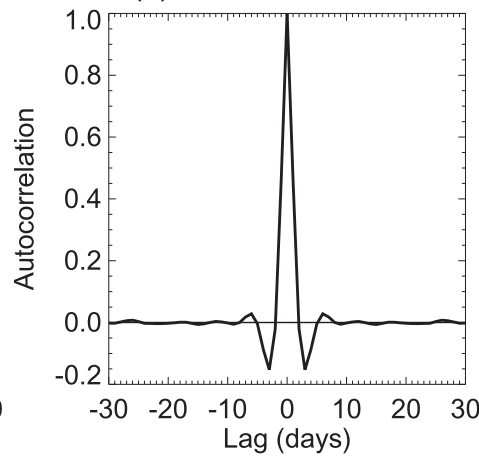

(e) $d\left(u_{s}\right.$ autocorrelation $) / d$ lag

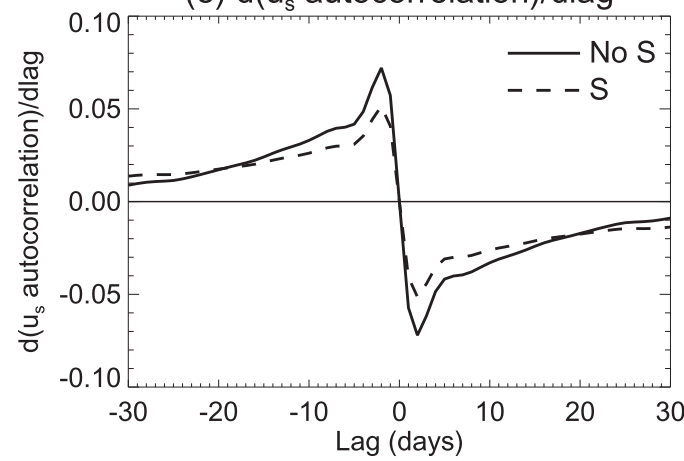

(g) Inferred feedback strength, b

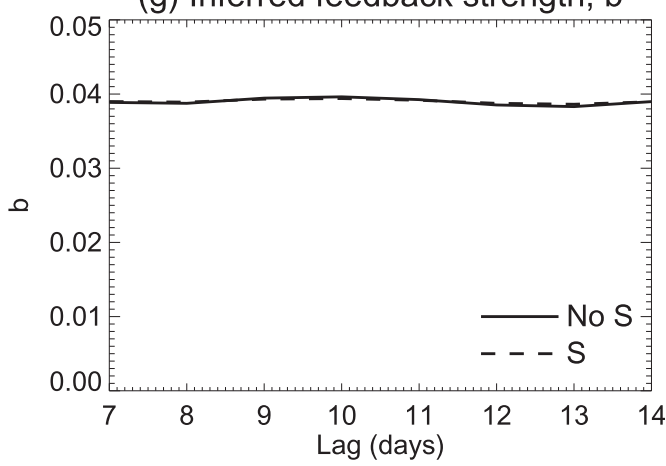

FIG. A1. (a) A subsection of the synthetic random eddy forcing time series $\tilde{m}$ generated using the AR2 process described in the appendix. (b) A subsection of the synthetic intraseasonal forcing time series $S$ described in the appendix. (c) The autocorrelation function of the eddy forcing time series shown in (a). (d) The autocorrelation function of the SAM zonal wind anomalies generated using (A2) (solid line) and (A3) (dashed line). (e)-(g) As in (d), but for the rate of change of the $u_{s}$ autocorrelation as a function of lag, the cross correlation between $m$ and $u_{s}$, and the calculated feedback strength $b$, respectively.

without any intraseasonal forcing, the correct value of 0.04 is obtained. However, for the time series where we have added the intraseasonal forcing $S$, a value of 0.053 is obtained, which we know to be incorrect since the time series has been constructed using $b=0.04$.

To get around this, our new method no longer depends on the cross correlation between $[\bar{u}]_{s}$ and $m$. Instead, we continue to assume the linear model of the feedback $m=\tilde{m}+b[\bar{u}]_{s}$ and isolate the feedback component of the eddy forcing by examining quantities regressed onto the SAM index as a function of lag. Regressing $m$ and $[\bar{u}]_{s}$ onto the SAM index (PC, which in this case is $[\bar{u}]_{s}$ as we are working with dimensionless time series) we obtain regression coefficients at lag $l$, $\beta_{m}(l)$ and $\beta_{u_{s}}(l)$, such that $m(t+l) \approx \beta_{m}(l) \mathrm{PC}(t)$ and $u_{s}(t+l) \approx \beta_{u_{s}}(l) \mathrm{PC}(t)$. At sufficiently large lags, beyond the autocorrelation time scale of the random eddy forcing $\tilde{m}$ the regressed component of the random part of the eddy forcing should be zero since it is not being organized by the SAM in any way and the component that 
shows up in the $m$ regression is solely the eddy feedback component [i.e., $\left.b u_{s}(t+l) \approx \beta_{m}(l) \mathrm{PC}(t)\right]$. Therefore, we have $b u_{s}(t+l) \approx \beta_{m}(l) \mathrm{PC}(t)$ and $u_{s}(t+l) \approx \beta_{u_{s}}(l) \mathrm{PC}(t)$. The feedback strength can therefore be obtained by

$$
b=\frac{\beta_{m}}{\beta_{u_{s}}} .
$$

This ratio is shown for both synthetic time series in Fig. A1g between lags of +7 and +14 days and it can be seen that for both time series, the feedback estimate is $b \approx 0.04$, as desired.The $95 \%$ confidence intervals on the feedback strength were obtained using a bootstrapping with replacement resampling technique. When considering a season of length $n d$ days with $n y$ years of simulation/reanalysis, there are $n=n d \times n y$ days of $\mathrm{PC}(t)$. To obtain an uncertainty estimate on $b, \mathrm{PC}(t)$ is resampled randomly with replacement from the sample of length $n$ to obtain a new time series of PC of length $n$. The corresponding $[\bar{u}]_{S}$ and $m$ data at lag $l$ from the new PC points are used to obtain regression coefficients $\beta_{u_{s}}(l)$ and $\beta_{m}(l)$ for this new time series. This was done 1000 times to obtain 1000 estimates of $\beta_{u_{s}}(l)$ and $\beta_{m}(l)$. The minimum and maximum estimates of these regression coefficients at $95 \%$ confidence were then obtained from this bootstrapping sample and the minimum and maximum estimates of $b$ that these gave are taken to be the $95 \%$ confidence interval on $b$.

\section{REFERENCES}

Arakelian, A., and F. Codron, 2012: Southern Hemisphere jet variability in the IPSL GCM at varying resolutions. J. Climate, 69, 3788-3799.

Baldwin, M. P., D. B. Stephenson, D. W. J. Thompson, T. J. Dunkerton, A. J. Charlton, and A. O'Neill, 2003: Stratospheric memory and skill of extended-range weather forecasts. Science, 301, 636-640, doi:10.1126/science.1087143.

,-- , and I. T. Jolliffe, 2009: Spatial weighting and iterative projection methods for EOFs. J. Climate, 22, 234-243.

Barnes, E. A., and D. L. Hartmann, 2010: Dynamical feedbacks of the southern annular mode in winter and summer. J. Atmos. Sci., 67, 2320-2330.

,-- D. M. W. Frierson, and J. Kidston, 2010: Effect of latitude on the persistence of eddy-driven jets. Geophys. Res. Lett., 37, L11804, doi:10.1029/2010GL043199.

Dee, D. P., and Coauthors, 2011: The ERA-Interim reanalysis: Configuration and performance of the data assimilation system. Quart. J. Roy. Meteor. Soc., 137, 553-597, doi:10.1002/qj.828.

Devore, J. L., 1999: Probability and Statistics for Engineering and the Sciences. 5th ed. Brooks/Cole, 535-536.

Ding, Q., E. L. Steig, D. S. Battisti, and J. M. Wallace, 2012: Influence of the tropics on the southern annular mode. J. Climate, 25, 6330-6348.

Feldstein, S., and S. Lee, 1998: Is the atmospheric zonal index driven by an eddy feedback? J. Atmos. Sci., 55, 3077-3086.

Gerber, E. P., L. M. Polvani, and D. Ancukiewicz, 2008: Annular mode time scales in the Intergovernmental Panel on Climate
Change Fourth Assessment Report models. Geophys. Res. Lett., 35, L22707, doi:10.1029/2008GL035712.

and Coauthors, 2010: Stratosphere-troposphere coupling and annular mode variability in chemistry-climate models. J. Geophys. Res., 115, D00M06, doi:10.1029/2009JD013770.

Kidson, J. W., 1988: Indices of the Southern Hemisphere zonal wind. J. Climate, 1, 183-194.

Kidston, J., and E. P. Gerber, 2010: Intermodel variability of the poleward shift of the austral jet stream in the CMIP3 integrations linked to biases in 20th century climatology. Geophys. Res. Lett., 37, L09708, doi:10.1029/2010GL042873.

— D. M. W. Frierson, J. A. Renwick, and G. J. Vallis, 2010: Observations, simulations, and dynamics of jet stream variability and annular modes. J. Climate, 23, 6186-6199.

Kuroda, Y., and H. Mukougawa, 2011: Role of medium-scale waves on the southern annular mode. J. Geophys. Res., 116, D22107, doi:10.1029/2011JD016293.

Leith, C. E., 1975: Climate response and fluctuation dissipation. J. Atmos. Sci., 32, 2022-2026.

L'Heureux, L. M., and D. W. J. Thompson, 2006: Observed relationships between the El Niño-Southern Oscillation and the extratropical zonal-mean circulation. J. Climate, 19, 276-287.

Lorenz, D. J., and D. L. Hartmann, 2001: Eddy-zonal flow feedback in the Southern Hemisphere. J. Atmos. Sci., 58, 3312-3327. , and — 2003: Eddy-zonal flow feedback in the Northern Hemisphere winter. J. Climate, 16, 1212-1227.

McLandress, C., T. G. Shepherd, J. F. Scinocca, D. A. Plummer, M. Sigmond, A. I. Jonsson, and M. C. Reader, 2011: Separating the dynamical effects of climate change and ozone depletion. Part II: Southern Hemisphere troposphere. J. Climate, 24, 1850-1868.

Robinson, W. A., 1991: The dynamics of the zonal index in a simple model of the atmosphere. Tellus, 43A, 295-305.

_ 1996: Does eddy feedback sustain variability in the zonal index? J. Atmos. Sci., 53, 3556-3569.

_ 2000: A baroclinic mechanism for the eddy feedback on the zonal index. J. Atmos. Sci., 57, 415-422.

Scinocca, J. F., N. A. McFarlane, M. Lazare, J. Li, and D. Plummer, 2008: Technical note: The CCCma third generation AGCM and its extension into the middle atmosphere. Atmos. Chem. Phys., 8, 7055-7074.

Shepherd, T. G., 1987a: Rossby waves and two-dimensional turbulence in a large-scale zonal jet. J. Fluid Mech., 183, 467-509. 1987b: A spectral view of nonlinear fluxes and stationarytransient interaction in the atmosphere. J. Atmos. Sci., 44, 1168-1178.

Simpson, I. R., P. Hitchcock, T. G. Shepherd, and J. F. Scinocca, 2011: Stratospheric variability and tropospheric annular mode timescales. Geophys. Res. Lett., 38, L20806, doi:10.1029/ 2011 GL049304.

, M. Blackburn, and J. D. Haigh, 2012: A mechanism for the effect of tropospheric jet structure on the annular mode-like response to stratospheric forcing. J. Atmos. Sci., 69, 2152-2170.

, P. Hitchcock, T. G. Shepherd, and J. F. Scinocca, 2013: Southern annular mode dynamics in observations and models. Part I: The influence of climatological zonal wind biases in a comprehensive GCM. J. Climate, 26, 3953-3967.

Son, S., and Coauthors, 2010: The impact of stratospheric ozone on the Southern Hemisphere circulation changes: A multimodel assessment. J. Geophys. Res., 115, D00M07, doi:10.1029/2010JD014271.

Thompson, D. W. J., and J. M. Wallace, 2000: Annular modes in the extratropical circulation. Part I: Month-to-month variability. J. Climate, 13, 1000-1016. 